\title{
Stochastic approach to diffusion inside the chaotic layer of a resonance
}

\author{
Martín F. Mestre,,${ }^{1, *}$ Armando Bazzani, ${ }^{2}$ Pablo M. Cincotta, ${ }^{1}$ and Claudia M. Giordano ${ }^{1}$ \\ ${ }^{1}$ Grupo de Caos en Sistemas Hamiltonianos, Facultad de Ciencias Astronómicas y Geofísicas, UNLP, Argentina \\ and Instituto de Astrofísica de La Plata (CCT La Plata - CONICET, UNLP), Argentina \\ ${ }^{2}$ Dipartimento di Fisica e Astronomia, Università di Bologna, Italia and INFN sezione di Bologna, Italia \\ (Received 25 February 2013; revised manuscript received 6 November 2013; published 13 January 2014)
}

\begin{abstract}
We model chaotic diffusion in a symplectic four-dimensional (4D) map by using the result of a theorem that was developed for stochastically perturbed integrable Hamiltonian systems. We explicitly consider a map defined by a free rotator (FR) coupled to a standard map (SM). We focus on the diffusion process in the action $I$ of the FR, obtaining a seminumerical method to compute the diffusion coefficient. We study two cases corresponding to a thick and a thin chaotic layer in the SM phase space and we discuss a related conjecture stated in the past. In the first case, the numerically computed probability density function for the action $I$ is well interpolated by the solution of a Fokker-Planck (FP) equation, whereas it presents a nonconstant time shift with respect to the concomitant FP solution in the second case suggesting the presence of an anomalous diffusion time scale. The explicit calculation of a diffusion coefficient for a 4D symplectic map can be useful to understand the slow diffusion observed in celestial mechanics and accelerator physics.
\end{abstract}

DOI: 10.1103/PhysRevE.89.012911

PACS number(s): 05.45.Pq, 05.10.Gg, 05.60.Cd

\section{INTRODUCTION}

Diffusion in Hamiltonian systems with more than 2 degrees of freedom (DoF) is a long-standing open problem whose understanding is relevant to model the slow diffusion phenomena observed in physical systems well described by conservative deterministic differential equations. Reference [1] explains the concept of chaotic diffusion from the point of view of celestial mechanics and gives examples of this relevant process for the dynamics of small bodies of the solar system. Reference [2] gives further evidence of the fact that chaotic diffusion is an important element of the long-term dynamics of the asteroid belt and shows how simple models of chaotic diffusion can be used to estimate the age of asteroid families. Reference [3] makes a statistical study of the stability of the solar system by computing probability density functions (PDFs) for the eccentricity and inclination of the planets over 5 Gyr. Further applications of chaotic diffusion in the celestial mechanics setting are described in Refs. [4,5]. Another relevant application is the transverse diffusion in circular accelerators when one takes into account the multipolar components of the magnetic field which limit the dynamics aperture [6]. The coupling of the transverse phase space with longitudinal particle dynamics, the presence of scattering, and parametric dependence due to supplied current modulations (ripples) have been proposed as possible causes of the particle diffusion [7]. A possible explanation of the underlying diffusion mechanism is the simultaneous presence of a stochastic or chaotic perturbation and the nonlinear terms [8]. Reference [9] carries out a numerical computation of the slow diffusion of orbits through thin chaotic layers of a four-dimensional (4D) symplectic map that models accelerator dynamics. Recently, experiments have been performed to measure the beam diffusion rate in proton colliders [10], and an explicit calculation of the diffusion coefficients for $4 \mathrm{D}$ stochastically perturbed maps is needed to analyze the experimental data.

\footnotetext{
*mmestre@fcaglp.unlp.edu.ar
}

In the case of nearly integrable Hamiltonian systems, the interest is focused in the action variables. Depending on the strength of the perturbation, there are restrictions to the topology of the region of action space in which global diffusion can take place. In Refs. [11-14], the concept of Arnold diffusion (AD) is understood as the diffusion that takes place along the Arnold web ${ }^{1}$ of a system that satisfies simultaneously the hypothesis of the KAM [15] and the Nekhoroshev [11] theorems. In this sense, Refs. [13,16-20] show numerical evidence of $\mathrm{AD}$. For a particular type of systems, there are theorems focused in demonstrating analytically the existence of orbits which drift in action space a quantity of order one in a finite time interval. In particular, it has been proved [21] that a twist map, coupled to a map close to the antiintegrable limit, has many orbits that drift arbitrarily far. Their generic system has a phase space with a geometry similar to the one of the thick layer problem (see afterwards). Chirikov's theory [12] provides a formula to estimate quantitatively the value of the diffusion coefficient. It has been tested [22] in a particular 2.5 DoF nearly integrable Hamiltonian system, obtaining a good agreement between theory and experiments in a particular range of the size of the perturbation. The theory of Nekhoroshev $[11,23]$ gives upper (but not lower) bounds to the rate of $\mathrm{AD}$. The implications of this theory to the geometry and speed of $\mathrm{AD}$, in singly and multiply resonant domains in nearly integrable Hamiltonian systems, have recently been comprehensively reviewed by Refs. $[14,19]$. They show more light onto the connection between the diffusion coefficient and the size of the remainder of the optimal resonant normal form and propose an associated set of variables in which the $\mathrm{AD}$ can be visualized and measured, thus establishing a novel path for future stability and diffusion studies (e.g., $[20,24])$.

Another theoretical approach considers the stochastic pump $[25,26]$ model. Both references consider a $4 \mathrm{D}$ symplectic

\footnotetext{
${ }^{1}$ Arnold web is the intersection of all the perturbed resonant surfaces with the isoenergetic manifold.
} 
map whose phase space contains a thick and a thin chaotic layer, which correspond to overlapping and nonoverlapping regimes, respectively. In their model, the dynamics on the chaotic layer is responsible for the diffusion of a perturbed action associated to a libration regime. According to the width of the layer, they apply two analytical procedures to find that the diffusion coefficient is strongly dependent on the ratio of the characteristic frequencies of the chaotic layer and the librations. Nevertheless, due to the unavoidable approximations, this theory does not allow a detailed analysis of the dependence of the diffusion behavior on the local action. They perform numerical experiments to corroborate their results. References [27,28] verify this method in a 4D symplectic map that consists of two coupled standard maps (SM). Reference [28] also estimates the global rate of diffusion by weighting local diffusion rates with the relative volume occupied by the various chaotically accessible regions in the 4D phase space. This map has also been studied by Refs. [29,30], where the diffusion rate has been measured for different values of the coupling parameter. The definition of the diffusion coefficient used in [30] is not based in an ensemble average but in a time average of a single trajectory. For other dynamical and theoretical discussions, see Refs. [31-33].

The development of the stochastic dynamical systems theory [34] allows us to describe the diffusion in Hamiltonian systems by means of stochastic perturbations which mimic the chaotic dynamics [35-37] and to derive a Fokker-Planck (FP) equation for the PDF in the slow variables [38]. In some cases, it was possible to prove diffusive limit theorems for dynamical systems in the presence of (deterministic) chaotic perturbations letting that the amplitude of the perturbation tends to zero and the time to infinity [39]. According to this point of view, it is possible to justify averaging principles to prove the diffusive limit [40]. In Ref. [41], a FP equation for the PDF associated to the action variable of a 1.5 DoF Hamiltonian system is deduced, by means of the random phase approximation. This approximation is partially valid in regions with resonance overlap [12] and is based on the assumption that the mixing of the angle variables of a Hamiltonian system is sufficiently faster than the one of the action variables (quasilinear theory). In Ref. [42], there has been applied the quasilinear theory to another 1.5 DoF Hamiltonian system, obtaining a FP equation whose diffusion coefficient turns out to depend only on the action variable. Reference [43] presents a hierarchy of equations for the evolution of the PDF in the phase space of nearly integrable Hamiltonian systems of arbitrary dimension. In this method, the kinetic equation has timedependent coefficients, even in the case of an autonomous perturbation. This represents a major difference with respect to quasilinear theories. These equations have been tested numerically only in a 1.5 DoF Hamiltonian system. Reference [44] reviews many fractional kinetic models and their relationship with dynamical models, phase space topology, and other chaos characteristics, as Poincaré recurrences and sticky domains. Summing up, although there are previous works that model Hamiltonian diffusion with a FP equation, these are mainly focused in systems with less than $2 \mathrm{DoF}$
Reference [45] considers the following a priori unstable ${ }^{2}$ 4D symplectic map:

$$
\begin{aligned}
& I_{n+1}=I_{n}-\epsilon \sin \left(\theta_{n}+\psi_{n}\right), \\
& \theta_{n+1}=\theta_{n}+I_{n+1} \quad \bmod 2 \pi, \\
& J_{n+1}=J_{n}+K \sin \left(\psi_{n}\right)-\epsilon \sin \left(\theta_{n}+\psi_{n}\right), \\
& \psi_{n+1}=\psi_{n}+J_{n+1} \quad \bmod 2 \pi,
\end{aligned}
$$

where the variables $(I, \theta, J, \psi)$ and the parameters $(K, \epsilon)$ are dimensionless, as well as every other quantity used in this paper. For $\epsilon=0$ and $K \neq 0$, the map consists of two uncoupled two-dimensional (2D) maps: a free rotator in the $[I, \theta]$ plane and a SM of parameter $K$ in the $[J, \psi]$ plane. In Ref. [45], the authors show that in the case in which $\epsilon \neq 0$, the short-time correlations in the $[J, \psi]$ plane due to the chaotic layer affect the diffusion in the $[I, \theta]$ plane. They apply the characteristic function method [46] and find that the diffusion tensor depends on the parameters of the system but not on the action $(I)$. They make experiments for wide ranges of parameter values, finding agreement with predictions as long as $|K|>2$ and $|\epsilon|>2$. They remark that this system has two interesting limit situations: $|K| \gg 1,|\epsilon| \ll 1$ (thick layer) and $|K| \lesssim 1,|\epsilon| \ll 1$ (thin layer), both being out of reach of their method. Moreover, Ref. [47] presents numerical evidence of the fact that the correlation function of the increments $\Delta J_{n}$ of a SM with $K=3$ can be fitted (approximately) by an exponentially decaying oscillating function, which is the exact autocorrelation function of the stochastic rotator. The authors conjecture that if the chaotic movement of the $\psi$ variable is coupled with the dynamics of an integrable system, then it could be observed a diffusion in the phase space of the integrable system which is similar to the diffusion driven by a stochastic rotator.

In this paper, we will make a numerical application, on the map given by Eq. (1), of the averaging theorem discussed in [47] to be used in stochastically perturbed nearly integrable Hamiltonian systems, which generalizes the result [8]. We predict and numerically compute the diffusion coefficient associated to $I$ for two values of the parameter of the SM that correspond to the cases of thick and thin layer diffusion. We show that in the thick layer regime the PDF satisfies a FP equation in a "slow diffusion time," while in the thin layer regime the PDF presents a "time delay" with respect to the associated FP solution in the slow diffusion time, that could be related to a different scaling law between the real time and the diffusion time when the correlation of the chaotic perturbation is not decaying sufficiently fast. Moreover, we will review the mentioned conjecture.

In principle, our approach is applicable to any nonintegrable Hamiltonian system that can be, either locally or in the whole phase space, decomposed into an integrable and a chaotic system which are weakly coupled: e.g., the case of a perturbed simple nonlinear resonance. Implementation of the FP equation can facilitate the study of the parametric dependence on the diffusion process for a whole particle distribution.

\footnotetext{
${ }^{2}$ Following the definition given in Sec. 2 of Ref. [32].
} 
The structure of the article is as follows. In Sec. II, we will provide a short description of a version of the averaging theorem for stochastically perturbed integrable maps. In Sec. III, we will give an example of its use with harmonic noise. In Sec. IV, we will introduce a seminumerical method to compute the diffusion coefficient for the map (1). In Secs. V and VI, we will test the method, respectively, in the thick and thin layer regimes. Finally, in Sec. VII we provide the conclusions.

\section{AN AVERAGING THEOREM FOR STOCHASTIC SYSTEMS}

The first averaging theorem for a deterministic equation stochastically perturbed is established in Ref. [48]. Afterwards, in Ref. [8] this result is generalized proving that under certain conditions, a first integral of the unperturbed system weakly ${ }^{3}$ converges towards a diffusion process. This theorem can be extended to compute the diffusion limit of the dynamics of the actions of a stochastically perturbed Hamiltonian system (see [47] and references therein) and it has been applied in the case of an integrable Hamiltonian system perturbed by a stochastic rotator. There, the authors verify one of the conclusions of the theorem, which states that for small enough perturbations, the PDF of the action satisfies a FP equation whose diffusion coefficient depends on the correlation function of the stochastic process.

Reference [6] applies the theorem to a stochastically perturbed symplectic map. They show that in the limit of noise with small amplitude, a colored noise can excite a local diffusion of the action variable. In what follows, we restate the theorem, without demonstration.

Let $M: \mathbb{R}^{2} \rightarrow \mathbb{R}^{2}$ be a symplectic map with an elliptic fixed point in the origin and let $\mathcal{R}$ be a neighborhood of it that defines a stable region. Let us assume that in $\mathcal{R}$ the measure of the nonlinear resonances and of the chaotic regions is negligible so that it is possible to replace the original map by an integrable one $M_{0}: \mathbb{R}^{2} \rightarrow \mathbb{R}^{2}$. Moreover, let $\mathbf{x} \equiv(x, p) \in \mathbb{R}^{2}$.

Let $\xi_{n}$ be a stationary stochastic process, with zero mean value and unit variance, defined in some probability space associated to some sample space $\mathcal{S}$. Let $E[\ldots]$ denote the concomitant (theoretical) expectation value.

We consider a stochastic map $P_{n}$ of the following form:

$$
P_{n}(\mathbf{x})=\mathbf{x}+\epsilon \xi_{n} \mathbf{v}(\mathbf{x}),
$$

where $n \in \mathbb{N}$ and

$$
\mathbf{v}(\mathbf{x})=\left(\begin{array}{c}
0 \\
-\frac{d \tilde{V}(x)}{d x}
\end{array}\right)
$$

with $\tilde{V}(x)$ being a potential function.

Then, we will study the dynamics of the stochastically perturbed symplectic map:

$$
\mathbf{x}_{n+1}=P_{n} \circ M_{0}\left(\mathbf{x}_{n}\right),
$$

where "o" denotes the composition operation.

\footnotetext{
${ }^{3}$ Weak convergence implies convergence of the PDF.
}

Introducing the action-angle variables $(I, \theta)$ of the map $M_{0}$, the map (2) is rewritten as

$$
\left(\begin{array}{c}
\theta_{n+1} \\
I_{n+1}
\end{array}\right)=\exp \left(\epsilon \xi_{n} L_{V(I, \theta)}\right) \circ\left(\begin{array}{c}
\theta_{n}+\Omega\left(I_{n}\right) \\
I_{n}
\end{array}\right),
$$

where $V \equiv V(I, \theta)=\tilde{V}(x(I, \theta)), L_{V}$ is the Lie operator defined by the Poisson bracket $\{\ldots, V\}$ with the potential function, and $\Omega(I)$ is the frequency of $M_{0}$.

The potential admits a Fourier series development

$$
V(I, \theta)=\sum_{k} V_{k}(I) e^{i k \theta}
$$

For a fixed value of $\epsilon$, we introduce a frequency cutoff $k_{\text {max }}$ in the expansion (4) in order to neglect those terms with $\left\|V_{k}\right\| \leqslant$ $\epsilon$, where $\|\ldots\|$ denotes the supremum, or infinite, norm in $\mathcal{R}$.

The hypotheses of the theorem are the following:

(i) The unperturbed map is at least of class $\mathcal{C}^{2}$ in $\mathcal{R}$.

(ii) The noise $\xi_{n}$ satisfies a $\varphi$-mixing condition, i.e., if $f(x)$ and $g(x)$ are bounded measurable functions, then the following inequality holds:

$$
\left|E\left[f\left(\xi_{n}\right) g\left(\xi_{0}\right)\right]-E\left[f\left(\xi_{n}\right)\right] E\left[g\left(\xi_{0}\right)\right]\right| \leqslant\|f\|\|g\| \varphi(n),
$$

where the function $\varphi(n)$ is such that $\lim _{n \rightarrow \infty} n^{6} \varphi(n)=0$.

(iii) No resonance condition, of the form $k \Omega(I)-2 \pi q=0$ $(q \in \mathbb{Z})$ for $|k|<2 k_{\text {max }}(k \neq 0)$, is fulfilled in $\mathcal{R}$ when $V_{k}(I) \neq$ 0 in the expansion (4).

(iv) The following limit exists:

$$
\begin{aligned}
\mathcal{D}(I) \equiv & \lim _{N \rightarrow \infty} \frac{1}{N} \sum_{n=0}^{N-1} \sum_{m=0}^{N-1}\left\{\frac{\partial V}{\partial \theta_{0}}\left[I, \theta_{0}+\Omega(I) n\right]\right. \\
& \left.\times \frac{\partial V}{\partial \theta_{0}}\left[I, \theta_{0}+\Omega(I) m\right] E\left[\xi_{n} \xi_{m}\right]\right\},
\end{aligned}
$$

where

$$
\frac{\partial V}{\partial \theta_{0}}\left[I, \theta_{0}+\Omega(I) n\right] \equiv \frac{\partial V}{\partial \theta}(I, \theta)_{\mid \theta=\theta_{0}+\Omega(I) n} .
$$

The second assumption is a condition on the losing memory rate of the process $\xi_{n}$, and the function $\varphi(n)$ is a measure of the independence between past and future. This condition is necessary if one wants to approximate the action dynamics with a diffusion process. The third requisite avoids the appearance of resonances between the unperturbed motion and the deterministic component of the perturbation $V(I, \theta)$. Due to this, the diffusion coefficient turns out to be independent of the initial angle $\left(\theta_{0}\right)$.

Being fulfilled these conditions, the following thesis is valid. Introducing the slow time $L=\epsilon^{2} n$, in the limit $\epsilon \rightarrow 0$ the stochastic process $I_{\epsilon}(L) \equiv I\left(L / \epsilon^{2}\right)$ weakly converges in $\mathcal{R}$ towards a diffusion process $\hat{I}(L)$ whose PDF, $\rho(\hat{I}, L)$, satisfies a FP equation of the form

$$
\frac{\partial \rho}{\partial L}(\hat{I}, L)=\frac{1}{2} \frac{\partial}{\partial \hat{I}}\left\{\mathcal{D}(\hat{I}) \frac{\partial \rho}{\partial \hat{I}}(\hat{I}, L)\right\} .
$$

The concept of weak convergence must be understood as convergence of the PDFs (from now on abbreviated as distributions) for a sequence of stochastic processes. Let $\rho(I, 0)$ be the initial particle distribution and let $\rho_{\epsilon}(I, t)$ be 
its average evolution at a time $t$ according to the stochastic map (3) for a finite value of $\epsilon$. The following limit is valid:

$$
\lim _{\epsilon \rightarrow 0} \rho_{\epsilon}\left(I, L / \epsilon^{2}\right)=\rho(I, L),
$$

where $\rho(I, L)$ is the solution of the FP equation (6). This limit is the definition of convergence of the distribution. Therefore, Eq. (6) can be used to get an approximation of the true distribution function. Under this point of view, $\mathcal{D}(I)$ is a rescaled diffusion coefficient. Taking into account the Fourier cutoff, Ref. [6] proves that the analytical diffusion coefficient, given by

$$
\mathcal{D}_{a}(I) \equiv \sum_{k}^{|k| \leqslant k_{\max }} k^{2}\left|V_{k}(I)\right|^{2} \tilde{\phi}[k \Omega(I)],
$$

is valid up to $\mathcal{O}(\epsilon)$, where the spectral density of the noise

$$
\tilde{\phi}(v)=\sum_{j=-\infty}^{\infty} \phi(|j|) e^{i j v}
$$

and the autocorrelation function

$$
\phi(n, m) \equiv E\left[\xi_{n} \xi_{m}\right]-E\left[\xi_{n}\right] E\left[\xi_{m}\right]=E\left[\xi_{n} \xi_{m}\right]
$$

have been introduced. Due to the fact that the noise is stationary, we have that $\phi(n, m)=\phi(n-m, 0) \quad \forall n, m \in \mathbb{N}$, so that in some opportunities we will use the notation $\phi(n) \equiv$ $\phi(n, 0)$.

Reference [47] remarks the importance of the spectral density in the behavior of the diffusion coefficient. $\tilde{\phi}(v)$ contributes to $\mathcal{D}_{a}(I)$ only with the amplitudes of the frequencies $v=k \Omega(I)$ which enter in the Fourier expansion (4) of the perturbation. As a consequence, the diffusion is enhanced when the spectral density of the noise is peaked at the frequencies $k \Omega(I)$.

Let us explain this issue considering a simple situation in which the spectral density is zero except in a neighborhood of radius $\Delta v$ of a fixed value $v_{0}$ :

$$
\tilde{\phi}(v)= \begin{cases}1 & \text { if }\left|v-v_{0}\right|<\Delta v, \\ 0 & \text { if }\left|v-v_{0}\right| \geqslant \Delta v .\end{cases}
$$

Thus, $\mathcal{D}_{a}(I)$ will be different from zero only in those actions that satisfy simultaneously that $v_{0}-\Delta v<k \Omega(I)<v_{0}+\Delta v$ and $V_{k}(I) \neq 0$. If we also assume that $\Omega$ has an inverse function, denoted by $\Omega^{-1}$, it can be said that $\mathcal{D}_{a}(I)$ will be different from zero around the action values $I=\Omega^{-1}\left(v_{0} / k\right)$.

\section{AN EXAMPLE WITH A FREE ROTATOR PERTURBED BY COLORED GAUSSIAN NOISE}

In order to show how this approach can be applied, we consider the stochastic symplectic map

$$
\begin{aligned}
& I_{n+1}=I_{n}-\epsilon \frac{\partial H_{1}}{\partial \theta_{n}}\left(I_{n+1}, \theta_{n}, \xi_{n}\right), \\
& \theta_{n+1}=\theta_{n}+\Omega\left(I_{n+1}\right)=\theta_{n}+I_{n+1} \bmod 2 \pi,
\end{aligned}
$$

where

$$
H_{1}(I, \theta, \xi)=H_{1}(\theta, \xi)=\xi V(\theta)=\xi \cos \theta .
$$

In other words, our system is a free rotator, with angle $\theta$ and angular velocity $\Omega(I) \equiv I$, perturbed with a stochastic term.
As the Fourier series of our potential is

$$
V(\theta)=\cos (\theta)=\frac{1}{2} e^{i \theta}+\frac{1}{2} e^{-i \theta},
$$

we have that

$$
V_{k}(I)= \begin{cases}\frac{1}{2} & \text { if } k= \pm 1 \\ 0 & \text { if } k \neq \pm 1\end{cases}
$$

The existence of only two terms in Eq. (12) implies that it is not necessary to adopt a cutoff value. Besides, together with the third hypothesis and the fact that $\Omega(I)=I$, we have that

$$
\mathcal{R}=\{I \in \mathbb{R}: \quad I \neq 2 \pi q, \quad q \in \mathbb{Z}\} .
$$

Thus, the analytical expression (8) takes the form

$$
\mathcal{D}_{a}(I)=\frac{1}{4}\{\tilde{\phi}(-I)+\tilde{\phi}(I)\} .
$$

This analytical result will be compared with a numerically computed diffusion coefficient defined in terms of the variance (for $N$ units of time)

$$
\mathcal{D}_{n u}(I ; N) \equiv \frac{\left\langle\left(I_{N}-\left\langle I_{N}\right\rangle\right)^{2}\right\rangle}{\epsilon^{2} N},
$$

where $\langle\ldots\rangle$ denotes a numerical average on the noise realization. For a finite number of noise realizations $N_{r}$, the numerical average of an arbitrary quantity $A$ at time $t$ is given by

$$
\left\langle A_{t}\right\rangle \equiv \frac{1}{N_{r}} \sum_{k=1}^{N_{r}} A_{t}^{(k)},
$$

where $A_{t}^{(k)}$ stands for the value associated to the $k$ th realization. Throughout this article, we will use $N_{r}=10^{5}$.

We use a type of Gaussian colored noise which consists of an ensemble of damped stochastic harmonic oscillators. Following [49-53], the Langevin equation of a unit-mass damped Brownian particle subject to the force field of a harmonic oscillator is

$$
\frac{d^{2} \xi_{t}}{d t^{2}}=-\lambda \frac{d \xi_{t}}{d t}-\omega^{2} \xi_{t}+c^{1 / 2} \Gamma_{t},
$$

where $\omega, \lambda$, and $c$ are positive constants to be defined below and where $\Gamma_{t}$ denotes a normalized white-noise process, satisfying

$$
E\left[\Gamma_{t}\right]=0, \quad E\left[\Gamma_{t} \Gamma_{t^{\prime}}\right]=\delta\left(t-t^{\prime}\right) ; \quad \forall t, t^{\prime} .
$$

The constant $\omega$ denotes the deterministic frequency of the unperturbed $(\lambda=c=0)$ oscillator. The constant $\lambda$ denotes the friction coefficient whose inverse $\lambda^{-1}$ is a characteristic relaxation time.

For $\omega=\lambda=0, d \xi / d t$ is a Wiener process with diffusion coefficient $c$. Besides, this constant is related to the asymptotic diffusion coefficient $D_{B}$ that the Brownian particle would have in case of null potential $(\omega=0)$, like this [54]:

$$
D_{B}=c / 2 \lambda^{2} \text {. }
$$

The equation (16) can be explicitly solved [50,51], and assuming $\omega^{2}-\lambda^{2} / 4>0$, the analytic solution is

$$
\begin{aligned}
\xi_{t}= & \frac{\lambda \xi_{0}+2 v_{0}}{2 \omega_{1}} e^{-\frac{\lambda}{2} t} \sin \left(\omega_{1} t\right)+\xi_{0} e^{-\frac{\lambda}{2} t} \cos \left(\omega_{1} t\right) \\
& +\frac{\sqrt{c}}{\omega_{1}} \int_{0}^{t} e^{-\frac{\lambda}{2}(t-z)} \sin \left[\omega_{1}(t-z)\right] d W_{z},
\end{aligned}
$$




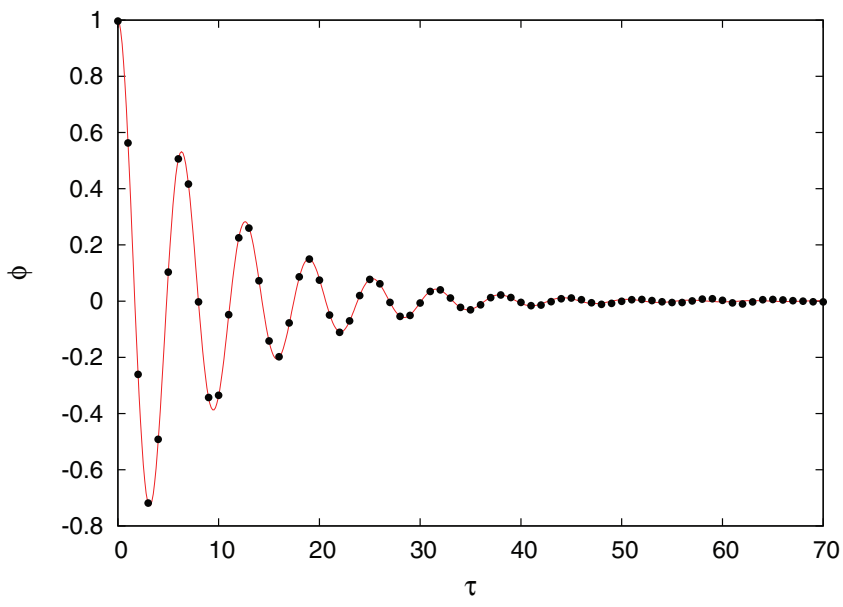

FIG. 1. (Color online) Autocorrelation function of an ensemble of damped stochastic harmonic oscillators for parameter values $\omega=1, \lambda=0.2$, and $c=0.4$ and for an initial condition given by $\left(\xi_{0}, v_{0}\right)=(-3,0)$. The asymptotic analytical function given in Eq. (18) is displayed with a red solid curve. The numerical function, for $N_{r}=10^{5}$ noise realizations and starting to measure since $t_{s}=$ 500 , is displayed with black dots. Here and in all the other figures, every plotted quantity is in dimensionless units.

where $\omega_{1} \equiv \sqrt{\omega^{2}-\lambda^{2} / 4}$ is the single proper frequency of the noise and where $d W_{z}$ is the differential of a Wiener process.

In this paper, we consider deterministic initial distributions and each realization corresponds to a realization of the Wiener process. The process tends asymptotically, for $t \rightarrow+\infty$, towards a stationary state with zero mean, with autocorrelation function (see Appendix A)

$$
\phi_{S}(\tau)=\frac{c}{2 \lambda \omega^{2}} e^{-\frac{\lambda}{2} \tau}\left\{\cos \left(\omega_{1} \tau\right)+\frac{\lambda}{2 \omega_{1}} \sin \left(\omega_{1} \tau\right)\right\},
$$

and with variance

$$
\sigma_{s}^{2}=\phi_{s}(0)=\frac{c}{2 \lambda \omega^{2}}
$$

We have performed a numerical stochastic integration for parameter values given by $\omega=1, \lambda=0.2$, and $c=$ 0.4 and for an initial condition given by $\left(\xi_{0}, v_{0}\right)=(-3,0)$. This corresponds to $\omega_{1} \approx 0.995$. We have used the SDERK ${ }^{4}$ integration package. It contains many schemes for obtaining strong solutions to stochastic differential equations. We have used one which consists of a fourth order Runge-Kutta scheme for the deterministic part and a first order for the stochastic part. We considered an ensemble of $N_{r}$ noise realizations during a total integration time of $t_{T}=10^{3}$. We have numerically estimated a relaxation time $t_{s}=500 \mathrm{such}$ that for $t>t_{s}$ the system can be considered in a stationary state.

Thus, in Fig. 1, we display $\phi\left(t_{s}, t_{s}+\tau\right)$, for $0 \leqslant \tau \leqslant 70$, with black dots. In the same figure, the analytic solution for $\phi_{s}(\tau)$ is illustrated with a red solid curve.

\footnotetext{
${ }^{4}$ Written by Daniel Steck, http://steck.us/computer.html
}

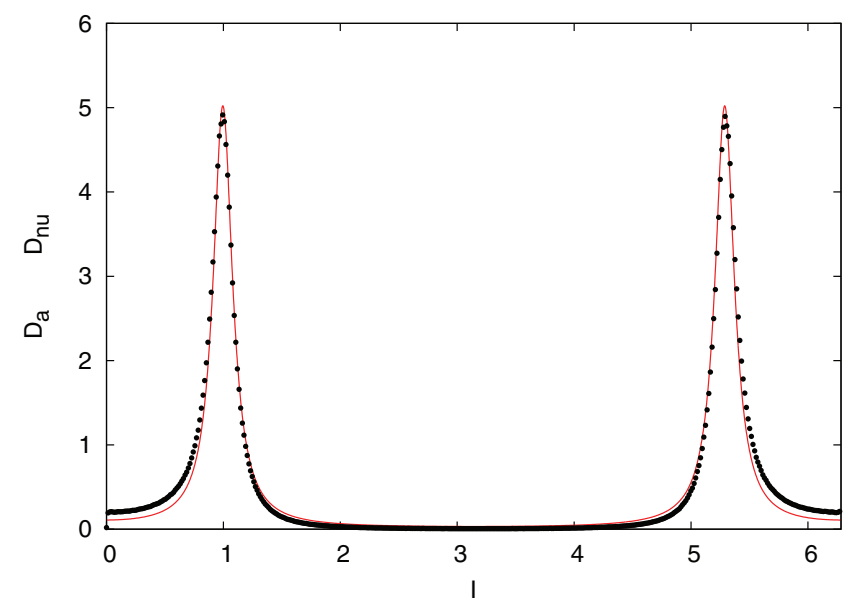

FIG. 2. (Color online) $\mathcal{D}_{a}(I)$ (red solid curve) and $\mathcal{D}_{n u}(I ; 500)$ (black dots) for the map given by Eq. (10). As noise we have used a stationary ensemble of damped harmonic oscillators with parameter values $\omega=1, \lambda=0.2$, and $c=0.4$ and with an initial condition given by $\left(\xi_{0}, v_{0}\right)=(-3,0)$. In the case of the numerical coefficient we have used $\epsilon=10^{-7}$, an initial angle given by $\theta_{0} \approx 0.214$ and the values of the same noise realizations computed for the previous figure, taking into account only the time interval: $t_{s} \leqslant t \leqslant 2 t_{s}\left(t_{s}=500\right)$.

The formula for the spectral density associated to this asymptotic state is given by Eqs. (B4), (B5), and (B6). Introducing it into Eq. (14), we have computed $\mathcal{D}_{a}(I)$ and displayed it in Fig. 2 with a red solid curve.

In the same figure, we display with black dots the quantity $\mathcal{D}_{n u}(I ; 500)$, for 500 values of $I$ placed equidistantly in the interval $[0,2 \pi)$, where we have used $\epsilon=10^{-7}$, an initial angle $\theta_{0} \approx 0.214$, and the values of the same noise realizations computed for the previous figure, taking into account only the time interval: $t_{s} \leqslant t \leqslant 2 t_{s}$. Throughout this article, we keep fixed the value $\theta_{0}$, unless explicitly stated otherwise. We can see that both coefficients agree for every action value and that the biggest difference takes place in the neighborhood of $I=0$ and $2 \pi$, which are points that are outside of the region $\mathcal{R}$ defined by Eq. (13). Moreover, we see that the diffusion coefficient reaches its maximum value for $I=\omega_{1}$ and $2 \pi-\omega_{1}$. This agrees with the analytical condition for diffusion enhancement that applies to this system: $\omega_{1}=$ $\pm \Omega(I)= \pm I(\bmod 2 \pi)$. We have carried out other numerical experiments changing $\epsilon$ and the result is similar as long as $\epsilon \lesssim 10^{-4}$.

Now, we point out a connection between the damped harmonic stochastic oscillator and the stochastic rotator. On the one hand, we have that, for the chosen parameter values of the harmonic noise, the contribution of the term with the sine function in $\phi_{s}$ is quite small, in such a way that neglecting this term hardly produces any change in $\mathcal{D}_{a}$. On the other hand, we know [47] that the exact analytical asymptotic autocorrelation function of the stochastic rotator is of the form $\phi_{s r}(\tau)=a e^{-b \tau} \cos \left(\omega_{\star} \tau\right)$, for some parameters $a, b$, and $\omega_{\star}$. Thus, we conclude that the diffusion coefficient produced by a coupling with a stochastic rotator is similar to the one produced by a coupling with the harmonic noise whenever the condition $\lambda \ll 2 \omega_{1}$ is satisfied. 
In the next section, we will discuss the stochastic approach to the computation of the diffusion coefficient in chaotic systems.

\section{A SEMINUMERICAL METHOD FOR CHAOTIC SYSTEMS}

In the previous sections, we have seen that the autocorrelation function of the noise has a net effect in the diffusion. In fact, the expression for $\mathcal{D}(I)$ depends on the trajectories of the integrable part and on the autocorrelation function of the noise and it is related with the Taylor-Green-Kubo formula (see [55] and references therein).

We wonder whether the hypothesis of the averaging theorem, whose result is the FP equation (6) with diffusion coefficient given by Eq. (8), could be modified in order to admit deterministic perturbations. We will show empirically that a particular chaotic perturbation can produce a diffusion process in the integrable part that can be modeled by the mentioned kinetic equation and will give a seminumerical method to estimate the diffusion coefficient.

In order to show how we intend to apply the stochastic formalism to a symplectic map with divided phase space, we will introduce two intermediate deterministic systems that link the one given by Eqs. (10) and (11) with the one given by Eq. (1), being the latter the main object of study of this article.

The first intermediate map is built by replacing, in Eq. (11), the stochastic process $\xi$ by an ensemble of chaotic trajectories. In particular, we set $\xi_{n}=\sin \left(\psi_{n}\right)$, where $\psi_{n}$ is the angle at time $n$ that corresponds to a chaotic trajectory of the SM. Thus, we have a (pseudo)sample space $\mathcal{S}$, which is some subset of the trajectories that belong to a particular chaotic layer of the SM. Due to the unicity of the solution of initial value problems for deterministic systems, it is possible to label $\mathcal{S}$ unambiguously with the values of the initial conditions in the $[J, \psi]$ plane. In Secs. V and VI, we will give explicit expressions for the sample spaces. Thus, we have the following skew coupled map:

$$
\begin{array}{rlr}
I_{n+1} & =I_{n}-\epsilon \sin \left(\psi_{n}\right) \sin \left(\theta_{n}\right), & \\
\theta_{n+1} & =\theta_{n}+I_{n+1} & \bmod 2 \pi, \\
J_{n+1} & =J_{n}+K \sin \left(\psi_{n}\right), & \\
\psi_{n+1} & =\psi_{n}+J_{n+1} \quad \bmod 2 \pi .
\end{array}
$$

The second intermediate map is constructed by generalizing the perturbation given in Eq. (11) to

$$
H_{1}(\theta, \psi)=\cos (\theta+\psi),
$$

obtaining the following (also partially coupled) map:

$$
\begin{array}{rlr}
I_{n+1} & =I_{n}-\epsilon \sin \left(\theta_{n}+\psi_{n}\right), & \\
\theta_{n+1} & =\theta_{n}+I_{n+1} & \\
J_{n+1} & =J_{n}+K \sin \left(\psi_{n}\right), & \\
\psi_{n+1} & =\psi_{n}+J_{n+1} \pi, &
\end{array}
$$

The statistical properties of hyperbolic maps are stable under small enough perturbations. Although the variables $(J, \psi)$ do not perform a hyperbolic dynamics, they have approximately this quality in any chaotic component of the SM.
We remark that the difference between the map (19) and the symplectic map (1) is the presence of feedback coupling between the slow dynamics and the chaotic dynamics $(J, \phi)$. In the case of strong chaos $K \gg 1$, a thick chaotic layer exists in the phase space and we do expect that the $\mathcal{O}(\epsilon)$ perturbation would not affect the diffusion process associated to the rotator action $I$. Conversely, in the case of weak chaos $K \simeq 1$, the presence of partial barriers in the phase space $(J, \psi)$ could be affected by a small perturbation even in the diffusion limit, and the statistical properties of the "noise" $\psi_{n}$ could depend on the long-time evolution so that the assumption of stationary noise can not be applied. However, we have numerically checked that the effect of this back coupling is negligible when computing diffusion coefficients for the slow variables $(I, \theta)$.

Thus, our idea is to model diffusion in the symplectic map (1) by applying a seminumerical scheme (related to the averaging theorem) to map (19). It can be demonstrated, following closely the proof in [6], that for a general perturbation of the form

$$
H_{1}(I, \theta, \psi)=\sum_{k} h_{k}(I) e^{i k(\theta+\psi)},
$$

the analytic diffusion coefficient is given by

$$
\mathcal{D}_{a}(I)=\sum_{k}^{|k| \leqslant k_{\max }} k^{2} h_{k}^{2}(I) \tilde{\phi}_{k, k}[k \Omega(I)], \quad \forall I \in \mathcal{R},
$$

where $\tilde{\phi}_{k, k}(v)$ are the spectral densities associated to the following autocorrelation functions of the SM: $\phi_{k, k^{\prime}}(n, m)=$ $E\left[e^{i k \psi_{n}} e^{i k^{\prime} \psi_{m}}\right]-E\left[e^{i k \psi_{n}}\right] E\left[e^{i k^{\prime} \psi_{m}}\right]$. As there are no analytical formulas for these autocorrelation functions valid for sufficiently long times, i.e., times of the order of 500, we will develop a seminumerical formula.

The generalization of the expression of $\mathcal{D}(I)$ given in Eq. (5), in the case of map (19), is

$$
\begin{aligned}
\mathcal{D}(I) \equiv & \lim _{N \rightarrow \infty} \frac{1}{N} \sum_{n=0}^{N-1} \sum_{m=0}^{N-1}\left\{E \left[\frac{\partial H_{1}}{\partial \theta_{0}}\left[\theta_{0}+\Omega(I) n, \psi_{n}\right]\right.\right. \\
& \left.\times \frac{\partial H_{1}}{\partial \theta_{0}}\left[\theta_{0}+\Omega(I) m, \psi_{m}\right]\right] \\
& -E\left[\frac{\partial H_{1}}{\partial \theta_{0}}\left[\theta_{0}+\Omega(I) n, \psi_{n}\right]\right] \\
& \left.\times E\left[\frac{\partial H_{1}}{\partial \theta_{0}}\left[\theta_{0}+\Omega(I) m, \psi_{m}\right]\right]\right\} .
\end{aligned}
$$

If instead of taking the limit, we evaluate numerically at a finite time $N$, we have the seminumerical diffusion coefficient

$$
\begin{aligned}
& \mathcal{D}_{s n}(I ; N) \\
& \equiv \frac{1}{N} \sum_{n=0}^{N-1} \sum_{m=0}^{N-1}\left\{\phi_{1}(n, m) \cos \left[\theta_{0}+\Omega(I) n\right] \cos \left[\theta_{0}+\Omega(I) m\right]\right. \\
& \quad+\phi_{2}(n, m) \sin \left[\theta_{0}+\Omega(I) n\right] \sin \left[\theta_{0}+\Omega(I) m\right] \\
& \quad+\phi_{3}(n, m) \cos \left[\theta_{0}+\Omega(I) n\right] \sin \left[\theta_{0}+\Omega(I) m\right] \\
& \left.\quad+\phi_{4}(n, m) \sin \left[\theta_{0}+\Omega(I) n\right] \cos \left[\theta_{0}+\Omega(I) m\right]\right\}
\end{aligned}
$$


where now the autocorrelation functions $\phi_{i}(i=1, \ldots, 4)$ should be computed numerically:

$$
\begin{aligned}
& \phi_{1}(n, m)=\left\langle\sin \psi_{n} \sin \psi_{m}\right\rangle-\left\langle\sin \psi_{n}\right\rangle\left\langle\sin \psi_{m}\right\rangle, \\
& \phi_{2}(n, m)=\left\langle\cos \psi_{n} \cos \psi_{m}\right\rangle-\left\langle\cos \psi_{n}\right\rangle\left\langle\cos \psi_{m}\right\rangle, \\
& \phi_{3}(n, m)=\left\langle\sin \psi_{n} \cos \psi_{m}\right\rangle-\left\langle\sin \psi_{n}\right\rangle\left\langle\cos \psi_{m}\right\rangle, \\
& \phi_{4}(n, m)=\left\langle\cos \psi_{n} \sin \psi_{m}\right\rangle-\left\langle\cos \psi_{n}\right\rangle\left\langle\sin \psi_{m}\right\rangle .
\end{aligned}
$$

For sufficiently large values of $N, \mathcal{D}_{s n}(I ; N)$ is effectively independent of $\theta_{0}$.

\section{THICK CHAOTIC LAYER}

In this section, we will test the stochastic approach to describe the diffusion in the symplectic map (1), using a parameter $K=3$. For this value, the measure of initial conditions in the phase space that give rise to chaotic orbits is much larger than the measure associated to regular orbits. The area of the islands of stability is small and almost every chaotic orbit belongs to the thick layer, also called chaotic sea. Moreover, the $J$ variable is unbounded for some trajectories so that the chaotic sea has an infinite extent. Notwithstanding, due to the $2 \pi$ periodicity of the perturbation functions, it is plausible to compactify $J$ to the interval $[0,2 \pi)$.

In order to compute $\mathcal{D}_{s n}(I, N)$, the first step is to compute the numerical autocorrelation functions $\phi_{i}$ defined in the previous section. Similarly to what was done in Sec. III, we select a time $n_{s}=500$, above which the ensemble of SM orbits are close to a stationary state (in the compactified phase space). This choice allows the ensemble to distribute all over the chaotic sea. As sample space we choose an ensemble of $N_{r}$ trajectories of the SM, with seeds placed along the straight line $J=J_{0} \equiv 3$ and considering only the time interval: $n_{s} \leqslant n \leqslant 2 n_{s}$. Let $\mathcal{S}_{n}$ be the ensemble of the sampled orbits at time $n$ :

$$
\begin{aligned}
\mathcal{S}_{n} \equiv & \left\{\left(J_{n}^{(k)}, \psi_{n}^{(k)}\right)=S^{n}\left[\left(J_{0}, u_{k}\right)\right]:\right. \\
& u_{k} \in \mathcal{U}(0,2 \pi) ; \\
k= & \left.1,2, \ldots, N_{r} ; \quad N_{r}=10^{5}\right\},
\end{aligned}
$$

where $S^{n}[\ldots]$ denotes $n$ iterations of the SM and $\mathcal{U}(a, b)$ denotes the uniform distribution in the segment $(a, b)$; we define $\mathcal{S}$ as the union of $\mathcal{S}_{n}$ with $n=n_{s}, n_{s}+1, \ldots, 2 n_{s}$ and $n_{s}=500$.

In Fig. 3, we can see the ensemble at both its initial state (seeds) $\mathcal{S}_{0}$ displayed with a black straight line, and at its nearly stationary state $\mathcal{S}_{n_{s}}$ displayed with dots.

In Fig. 4, we display, for $0 \leqslant \tau \leqslant 100$, the values of $\phi_{i}\left(n_{s}, n_{s}+\tau\right)$, for $i=1,2,3,4$, using black (square) dots, cyan (round) dots, blue (triangle-shaped) dots, and magenta (diamond-shaped) dots, respectively.

We notice that all of them oscillate in a neighborhood of zero, that $\phi_{1}$ presents oscillations of higher amplitude than the other cases, and that $\phi_{3}$ and $\phi_{4}$ have relatively small values at every moment. Besides, we appreciate that the autocorrelations do not decay to zero for $\tau \gg 1$, unlike what happened in the colored Gaussian case. In spite of this difference, there is a kind of similarity between $\phi_{s}$ and $\phi_{1}$ because a least squares fit of the ansatz $\phi_{1}(\tau)=\left(a_{0}+a e^{-b \tau}\right) \cos \left(\omega_{\star} \tau\right)$ (for parameters $a_{0}, a, b$, and $\left.\omega_{\star}\right)$ worked very well, even up to times of the

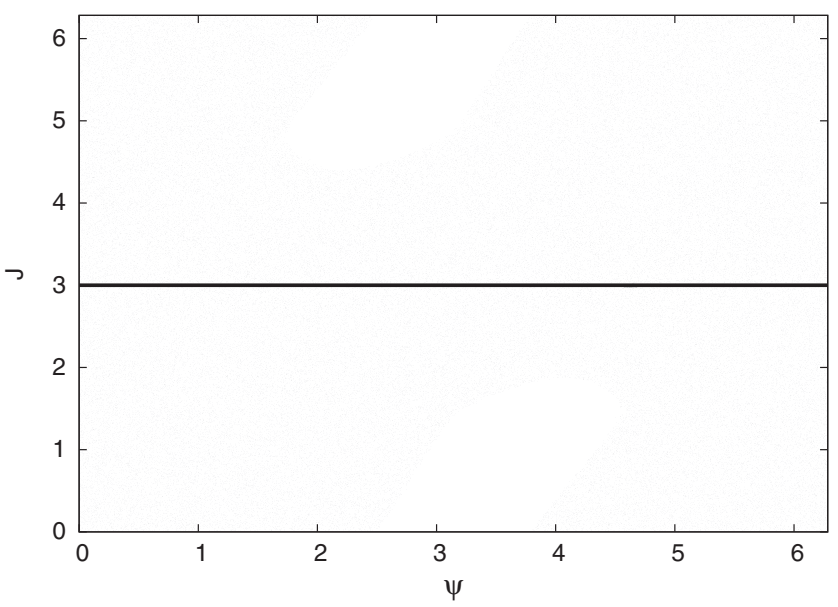

FIG. 3. Snapshots of the sample space associated to the SM with $K=3: \mathcal{S}_{0}$ is displayed with a black straight line while $\mathcal{S}_{n_{s}}$ ( $J$ compactified to the interval $[0,2 \pi)$ ) is displayed with dots.
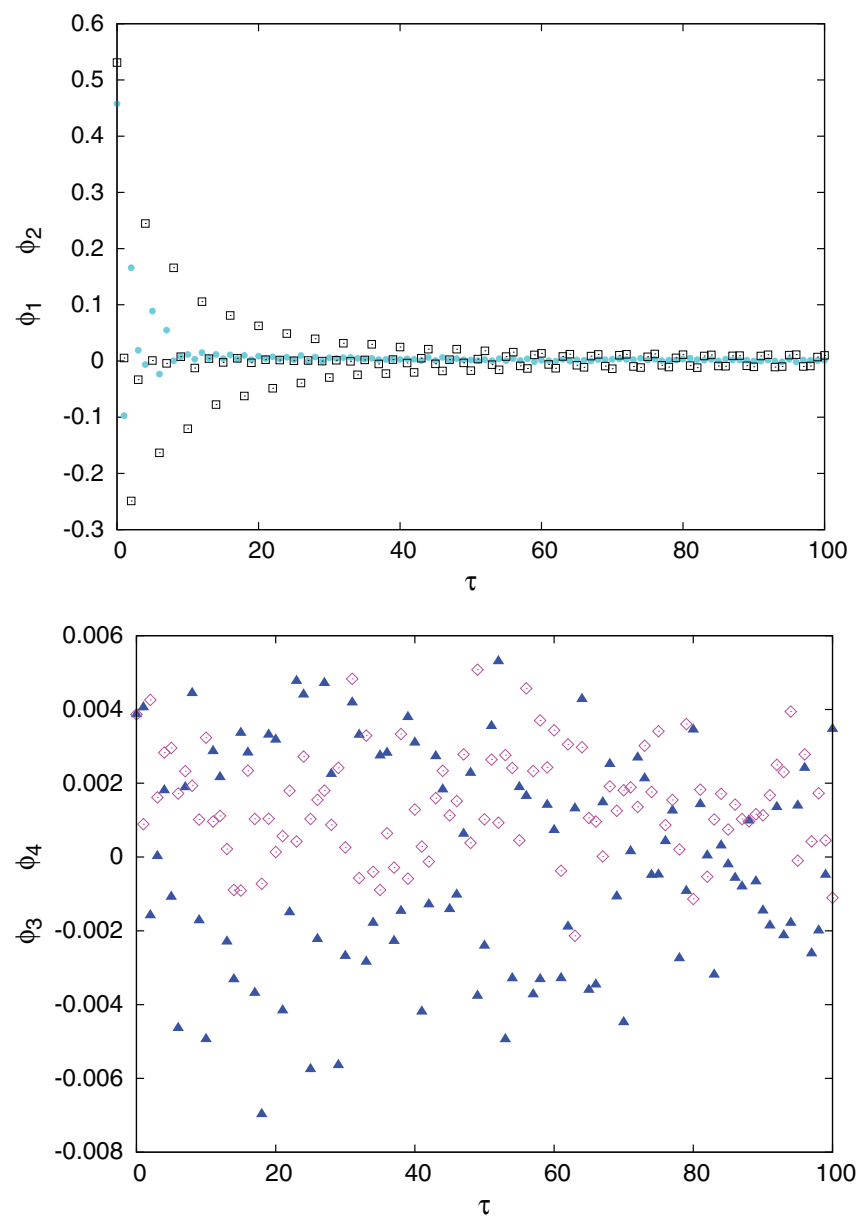

FIG. 4. (Color online) SM autocorrelation functions: $\phi_{i}\left(n_{s}, n_{s}+\right.$ $\tau)\left(n_{s}=500\right)$ of Eq. (22), for $i=1,2,3,4$, using black (square) dots, cyan (round) dots, blue (triangle-shaped) dots, and magenta (diamond-shaped) dots, respectively. The sample set is $\mathcal{S}=$ $\bigcup_{n=n_{s}}^{2 n_{s}} \mathcal{S}_{n}$, with $\mathcal{S}_{n}$ defined by Eq. (23). 

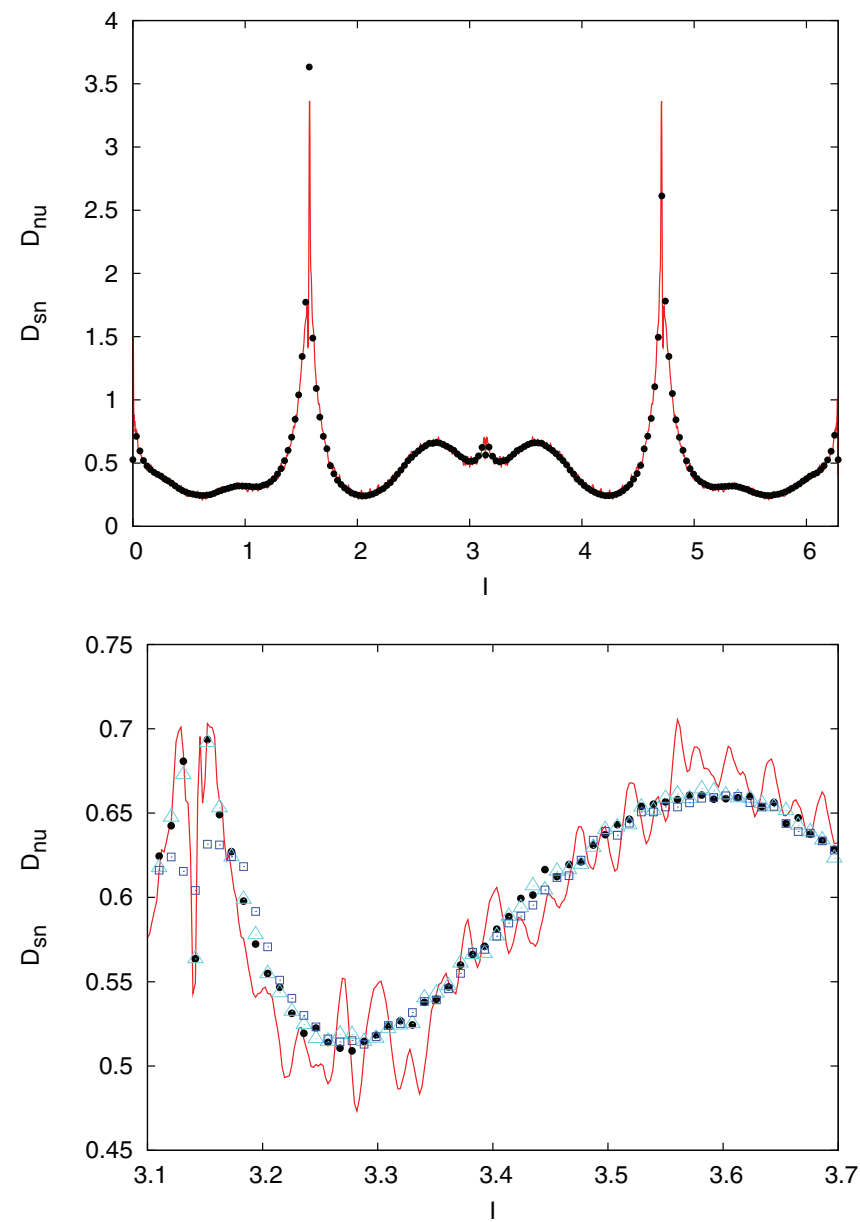

FIG. 5. (Color online) Diffusion coefficients for the symplectic map (1), using $K=3$ and $\mathcal{S}=\bigcup_{n=n_{s}}^{2 n_{s}} \mathcal{S}_{n}$ with $\mathcal{S}_{n}$ defined by Eq. (23). $\mathcal{D}_{s n}(I ; 500)$ is shown with a red solid line. $\mathcal{D}_{n u}(I ; 500)$ was computed for $\epsilon=10^{-3}, 10^{-5}, 10^{-7}$ and displayed with blue (square) dots, cyan (triangle-shaped) dots, and black (round) dots, respectively.

order of $\tau \sim 300$, giving a proper frequency $\omega_{\star} \approx 1.572 \approx$ $\pi / 2$.

We have introduced the previously computed four autocorrelation functions into Eq. $(21)$ in order to calculate $\mathcal{D}_{s n}(I ; 500)$ for 3000 equidistant values of $I$ in the interval $[0,2 \pi)$. In Fig. 5 (top), we compare this value with the numerical diffusion coefficient associated to the symplectic map (1) for $\epsilon=10^{-7}$, using a red solid curve and black (round) dots, respectively. For each value of $I$, the initial conditions of the ensemble chosen to compute $\mathcal{D}_{n u}(I, 500)$, according to Eq. (15), consist of the direct product between the point $\left(I, \theta_{0}\right)$ and $\mathcal{S}_{n_{s}}$.

The result is that $\mathcal{D}_{s n}$ succeeds in predicting $\mathcal{D}_{n u}$. In Fig. 5 (bottom), we display, for $3.1 \leqslant I \leqslant 3.7$, the same two quantities of the top panel together with $\mathcal{D}_{n u}(I ; 500)$ computed for $\epsilon=10^{-3}$ and $10^{-5}$ with blue (square) dots and cyan (triangle-shaped) dots, respectively.

We observe that the numerical coefficients for $\epsilon=10^{-5}$ and $10^{-7}$ are equivalent to each other throughout the interval whereas the one for $\epsilon=10^{-3}$ differs from the other coefficients in the neighborhood of $I=\pi$. This discrepancy is expected because the method is meant to work as long as $\epsilon$ is small enough, and it is in agreement with the result commented near the end of Sec. III.

Comparing the numerical diffusion coefficients of Figs. 2 and 5 (top), we see that the diffusion generated by coupling the free rotator with the SM is different from the one generated by coupling the same integrable system with an ensemble of damped stochastic harmonic oscillators. The only aspect in common between both coefficients is that they have absolute maximums in those actions whose associated frequency is equal to any of the characteristic frequencies of the perturbation (chaotic or stochastic). If we had used a harmonic noise with proper frequency similar to the one of the SM $\left(\omega_{1} \approx \pi / 2\right)$, we would have obtained a result analog to the one of Fig. 2 but with its maximums at $I \approx \pi / 2$ and $3 \pi / 2$. Taking this information into account together with the fact that the diffusion driven by a stochastic rotator is similar to the one driven by harmonic noise, we have given an example in which the effect of coupling an integrable map to a chaotic perturbation can not be modeled by the effect of coupling the same integrable map to a stochastic rotator (neither to a damped stochastic harmonic oscillator). Thus, we have refuted the conjecture cited in the Introduction.

Nevertheless, it is true that a chaotic perturbation can drive a process qualitatively and quantitatively similar to a diffusion process, as it will become clear in the rest of this section, where we will empirically show that the action $I$ of the 4D symplectic map (1) behaves as a diffusion process that satisfies the FP equation (6).

We have numerically solved the FP equation (6) for an ensemble with initial conditions following a Gaussian distribution, i.e., $I_{0} \sim \mathcal{N}(\mu, \sigma)$, with mean value $\mu=1.75$ and standard deviation $\sigma=0.1$. We used a Cranck-Nicholson implicit algorithm and the values of $\mathcal{D}(\hat{I})$ where those of $\mathcal{D}_{n u}(I ; 500)$, plotted in Fig. 5 (top). Using as input data $\mathcal{D}_{s n}$, instead of $\mathcal{D}_{n u}$, does not significantly change the results.

Besides, we have numerically computed the evolution of the symplectic map (1) of an ensemble of $N_{r}$ test particles with initial conditions such that

(i) $I_{0} \sim \mathcal{N}(1.75,0.1)$,

(ii) $\theta_{0}=(1+\sqrt{5}) / 2 \approx 1.618$,

(iii) $\left(J_{0}, \psi_{0}\right) \in \mathcal{S}_{n_{s}}$.

We used $\epsilon=10^{-3}$ so that the relation between the FP time $(L)$ and the (Hamiltonian) real time $(n)$ is

$$
L=\epsilon^{2} n=10^{-6} n \text {. }
$$

Figures 6 and 7 show both the FP solution $(\rho)$ and histograms done with the orbits of the symplectic map $\left(\rho_{\epsilon}\right)$. In the former figure, we have plotted the functions $\rho(I, 0)$, $\rho_{\epsilon}(I, 0), \rho(I, 0.02)$, and $\rho_{\epsilon}\left(I, 2 \times 10^{4}\right)$ in colors orange (smooth line A), green (histogram A), brown (smooth line B), and cyan (histogram B), respectively. It can be seen that the behavior of the action of the symplectic map (1) resembles closely the diffusion process given by the FP solution. The diffusion coefficient is displayed in the same figure, in order to show the reason why the left hand side of the initially Gaussian distribution diffuses faster than its right hand counterpart.

The second figure shows $\rho(I, 0.04), \rho_{\epsilon}\left(I, 4 \times 10^{4}\right)$, $\rho(I, 0.10), \rho_{\epsilon}\left(I, 10^{5}\right), \rho(I, 0.50)$, and $\rho_{\epsilon}\left(I, 5 \times 10^{5}\right)$ in colors orange (smooth line A), green (histogram A), brown (smooth 


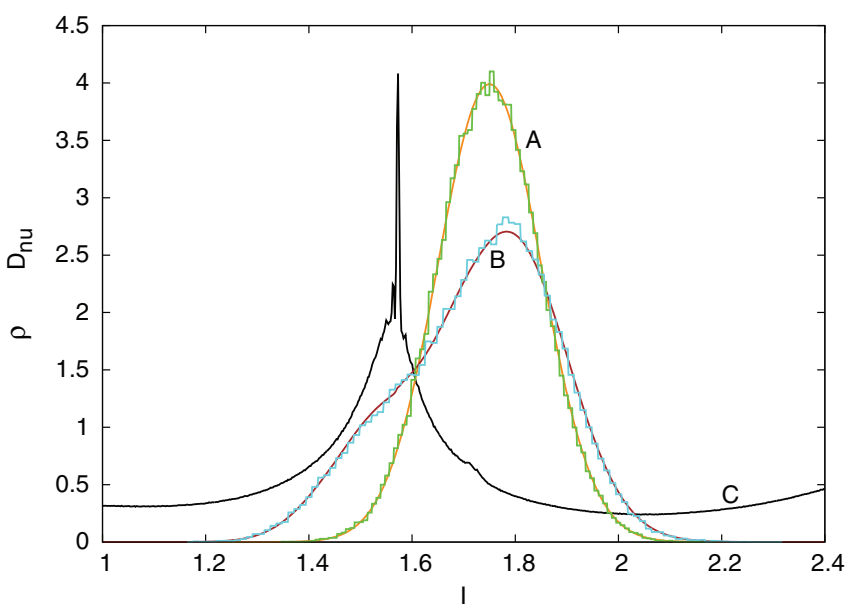

FIG. 6. (Color online) Distributions for thick layer regime $(K=$ 3): $\rho(I, 0), \rho_{\epsilon}(I, 0), \rho(I, 0.02)$, and $\rho_{\epsilon}\left(I, 2 \times 10^{4}\right)$ in colors orange (smooth line A), green (histogram A), brown (smooth line B) and cyan (histogram B), respectively. For the histograms, the coupling parameter is $\epsilon=10^{-3}$. The black line (C) displays $\mathcal{D}_{n u}(I ; 500)$, the diffusion coefficient used when integrating the FP equation.

line B), cyan (histogram B), magenta (smooth line C), and blue (histogram C), respectively.

To end up with the study of diffusion in the thick layer, we have computed the time evolution of the variance of the histograms for two values of the perturbation parameter. In Fig. 8, we show $\sigma^{2}(L)$, for $0 \leqslant L \leqslant 0.2$, for $\epsilon=10^{-3}$ and $10^{-4}$, respectively with round and square dots. We have used the variable $L$ in the temporal axis in order to have a time unit comparable for both $\epsilon$ values. More specifically, $L=0.2$ corresponds to $n=10^{6} \times 0.2=2 \times 10^{5}$ for $\epsilon=10^{-3}$, while it corresponds to $n=10^{8} \times 0.2=2 \times 10^{7}$ for $\epsilon=10^{-4}$. We observe that there is agreement between both variance evolutions.

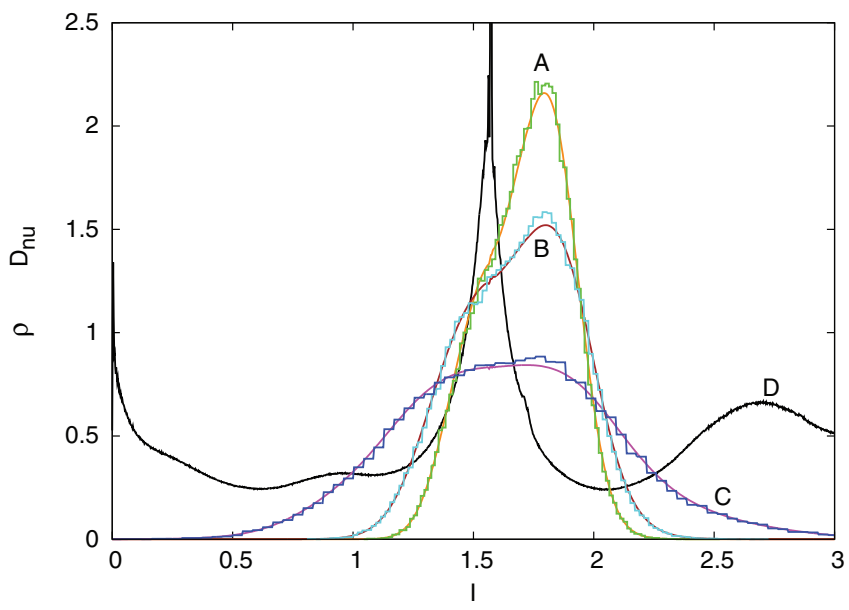

FIG. 7. (Color online) Distributions: $\rho(I, 0.04), \rho_{\epsilon}\left(I, 4 \times 10^{4}\right)$, $\rho(I, 0.10), \rho_{\epsilon}\left(I, 10^{5}\right), \rho(I, 0.50)$, and $\rho_{\epsilon}\left(I, 5 \times 10^{5}\right)$ in colors orange (smooth line A), green (histogram A), brown (smooth line B), cyan (histogram B), magenta (smooth line C), and blue (histogram C), respectively. For the histograms, the coupling parameter is $\epsilon=10^{-3}$. The black line (D) displays $\mathcal{D}_{n u}(I ; 500)$.

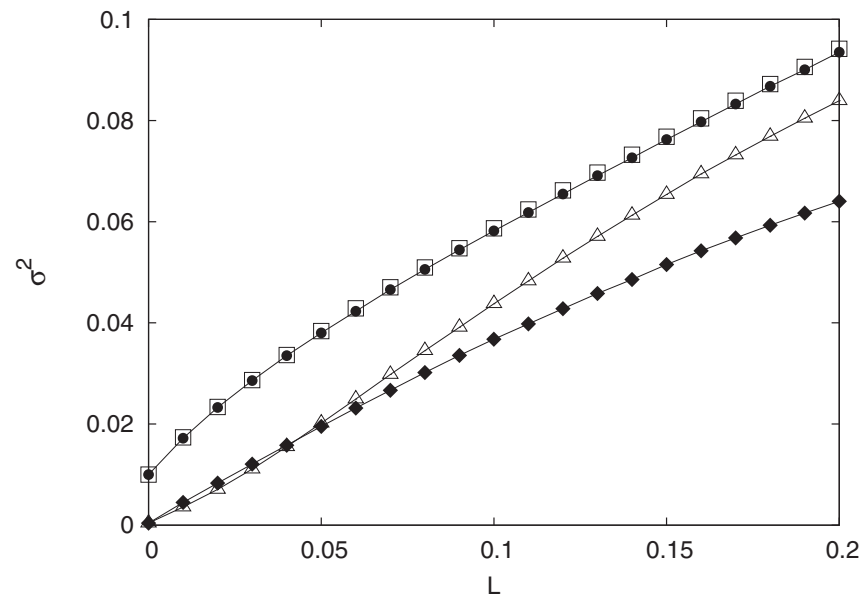

FIG. 8. $\sigma^{2}(L)$, for $0 \leqslant L \leqslant 0.2$. The round dots correspond to $K=3$ and $\epsilon=10^{-3}$, the square dots correspond to $K=3$ and $\epsilon=$ $10^{-4}$, the diamond-shaped dots correspond to $K=0.9$ and $\epsilon=10^{-3}$, and the triangle-shaped dots correspond to $K=0.9$ and $\epsilon=10^{-4}$.

The upper curve of Fig. 9 shows the same variance evolution, only for $\epsilon=10^{-3}$, for a bigger time interval: $0 \leqslant L \leqslant 2$. We observe an approximately linear behavior. This has been corroborated by making a least-squares fit of the ansatz $\sigma^{2}(L) \propto L^{w}$ and obtaining $w \approx 1.02$ for the time interval $0.5 \leqslant L \leqslant 2$. Thus, it can be said that under these circumstances, the global diffusion is highly close to normal.

In Fig. 10, we show a snaphshot of the phase space $(J, \psi)$ computed after $2 \times 10^{6}$ iterations (largest time used in our experiments with $\epsilon=10^{-3}$, corresponding to a diffusion time $L=2$ ), to be compared with Fig. 3 . The similarity of the two pictures suggests that the chaotic layer used to compute the diffusion coefficients is robust under the effect of $\mathcal{O}(\epsilon)$ perturbations even after relatively long times.

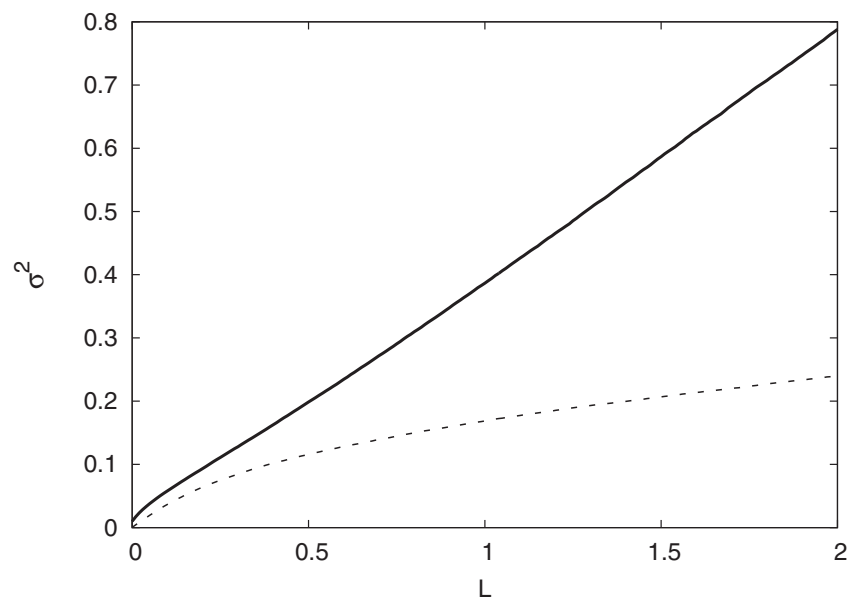

FIG. 9. $\sigma^{2}(L)$, for $0 \leqslant L \leqslant 2$ and $\epsilon=10^{-3}$. The upper curve corresponds to $K=3.0$ while the lower (dashed) curve corresponds to $K=0.9$. 


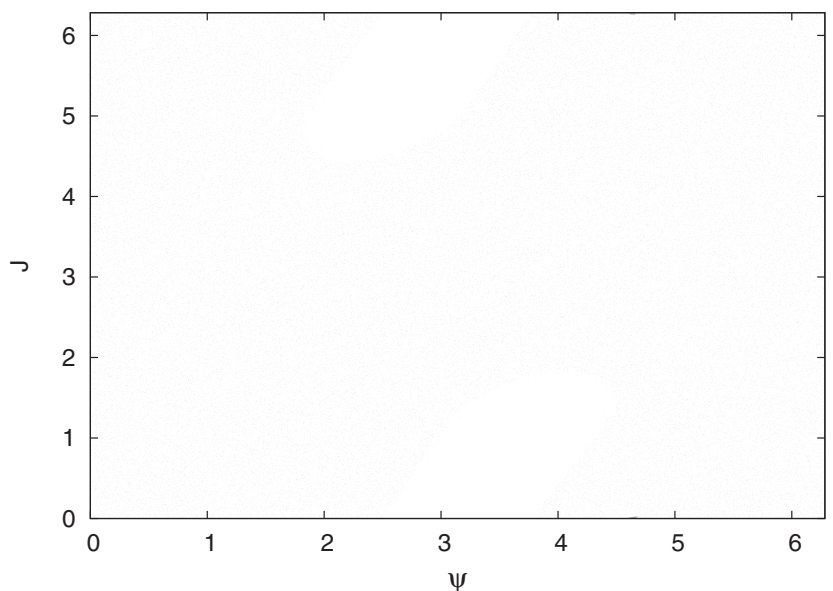

FIG. 10. Snapshot of the phase space $(J, \psi)$ for the map (1) computed after $2 \times 10^{6}$ iterations with $\epsilon=10^{-3}$ and $K=3$; i.e., projection onto the $[J, \psi]$ plane of the orbits associated to $\rho_{\epsilon}\left(I, 2 \times 10^{6}\right)$.

\section{THIN CHAOTIC LAYER}

In this section, we will work with a parameter $K=0.9$, following a similar procedure to that of the previous section. For this parameter value the area filled by islands of stability is considerably larger than before. In the phase space we have different separated chaotic layers, so that one has to choose the sample space more carefully than in the previous section.

In this opportunity, we choose the $N_{r}$ seeds of the SM to be placed along the segment defined by $J=J_{0} \equiv 0.5$ and $0.1 \leqslant \psi_{0} \leqslant 0.4$. Thus, we have a new sample space $\mathcal{S}$ defined by the union of the snapshots

$$
\begin{aligned}
\mathcal{S}_{n} \equiv & \left\{\left(J_{n}^{(k)}, \psi_{n}^{(k)}\right)=S^{n}\left[\left(J_{0}, u_{k}\right)\right]:\right. \\
& u_{k} \in \mathcal{U}(0.1,0.4) ; \\
k= & \left.1,2, \ldots, N_{r} ; \quad N_{r}=10^{5}\right\},
\end{aligned}
$$

for $n=n_{s}, n_{s}+1, \ldots, 2 n_{s}$ with $n_{s}=500$. Therefore, the sample space is the thin chaotic layer associated to the primary island chain $\left(J_{r}=0\right)$. Figure 11 displays $\mathcal{S}_{0}$ and $\mathcal{S}_{n_{s}}$ using a black line and dots, respectively.

In Fig. 12, we display, for $0 \leqslant \tau \leqslant 100$, the values of $\phi_{i}\left(n_{s}, n_{s}+\tau\right)$, for $i=1,2,3,4$, using black (square) dots, cyan (round) dots, blue (triangle-shaped) dots, and magenta (diamond-shaped) dots, respectively.

Even in this case the autocorrelations fluctuate around zero, and both $\phi_{3}$ and $\phi_{4}$ show the least amplitude of fluctuation. A distinctive characteristic is the fact that the amplitudes of $\phi_{1}(\tau)$ and $\phi_{2}(\tau)$ are similar to each other.

Figure 13 (top) shows, for $I \in[0,2 \pi)$, the seminumerical and the numerical diffusion coefficients, being the latter computed for $\epsilon=10^{-7}$. $\mathcal{D}_{s n}(I ; 500)$ and $\mathcal{D}_{n u}(I ; 500)$ are displayed using a red solid curve and black (round) dots, respectively. We notice that there is agreement between prediction and measurement, as in the $K=3$ case. Figure 13 (bottom) shows, for $5 \leqslant I \leqslant 6$, the same two quantities of the top panel together with $\mathcal{D}_{n u}(I ; 500)$ computed with $\epsilon=10^{-3}$ and $10^{-5}$, using blue (square) dots and cyan (triangle-shaped) dots, respectively. The result is analogous to the one obtained

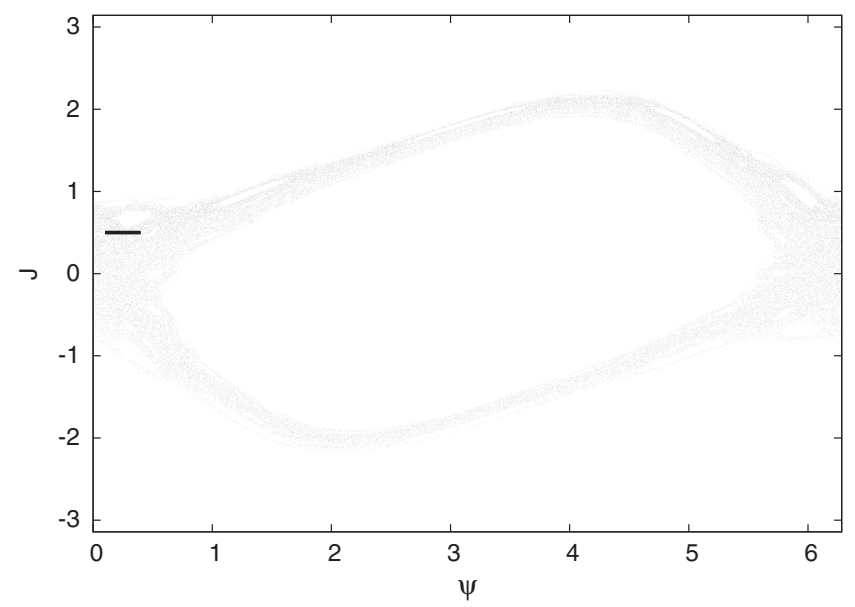

FIG. 11. Snapshots of the sample space associated to the SM with $K=0.9: \mathcal{S}_{0}$ is displayed with a black straight line while $\mathcal{S}_{n_{s}}$ is displayed with dots.

in Fig. 5 (bottom) regarding the fact that the method works better for the two smaller values of $\epsilon$.

This time we have solved numerically the FP equation with initial actions distributed according to a $\mathcal{N}(0.75,0.02)$ and the values of $\mathcal{D}(\hat{I})$ were those of $\mathcal{D}_{n u}(I ; 500)$, plotted in Fig. 13 (top). Besides, we have computed the evolution, for $\epsilon=10^{-3}$, of an ensemble of $N_{r}$ test particles with initial conditions given by

(i) $I_{0} \sim \mathcal{N}(0.75,0.02)$,

(ii) $\theta_{0}=(1+\sqrt{5}) / 2 \approx 1.618$,

(iii) $\left(J_{0}, \psi_{0}\right) \in \mathcal{S}_{n_{s}}$.

The orange (smooth line B) and green (histogram A) curves in Fig. 14 represent the functions $\rho(I, 0.03)$ and $\rho_{\epsilon}\left(I, 3 \times 10^{4}\right)$, respectively. If the relation between the $\mathrm{FP}$ and the symplectic times was the one given by Eq. (24), then we would have obtained that both functions match each other. Instead, we notice a difference: the FP solution is ahead of, i.e., more

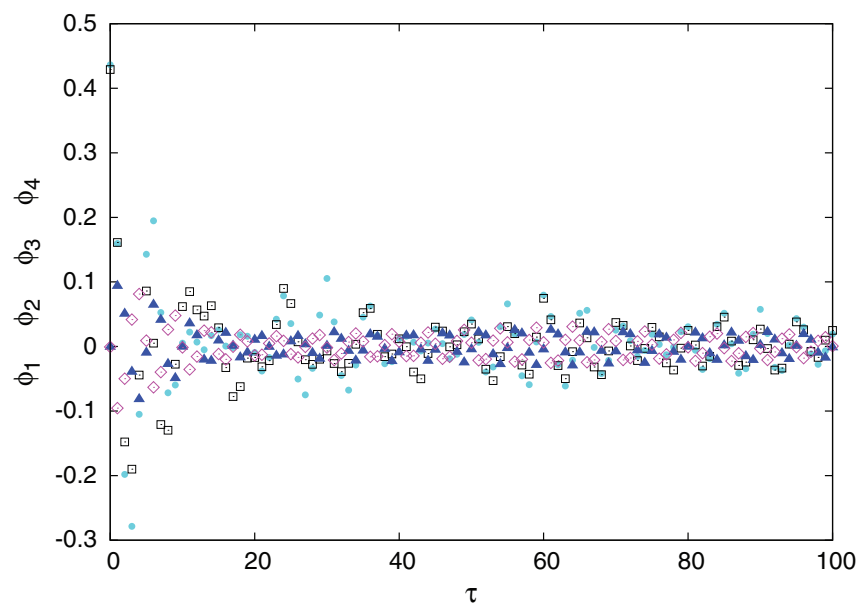

FIG. 12. (Color online) SM autocorrelation functions: $\phi_{i}\left(n_{s}, n_{s}+\tau\right) \quad\left(n_{s}=500\right)$ of Eq. (22), for $i=1,2,3,4$, using black (square) dots, cyan (round) dots, blue (triangle-shaped) dots, and magenta (diamond-shaped) dots, respectively. The sample set is $\mathcal{S}=\bigcup_{n=n_{s}}^{2 n_{s}} \mathcal{S}_{n}$, with $\mathcal{S}_{n}$ defined by Eq. (25). 

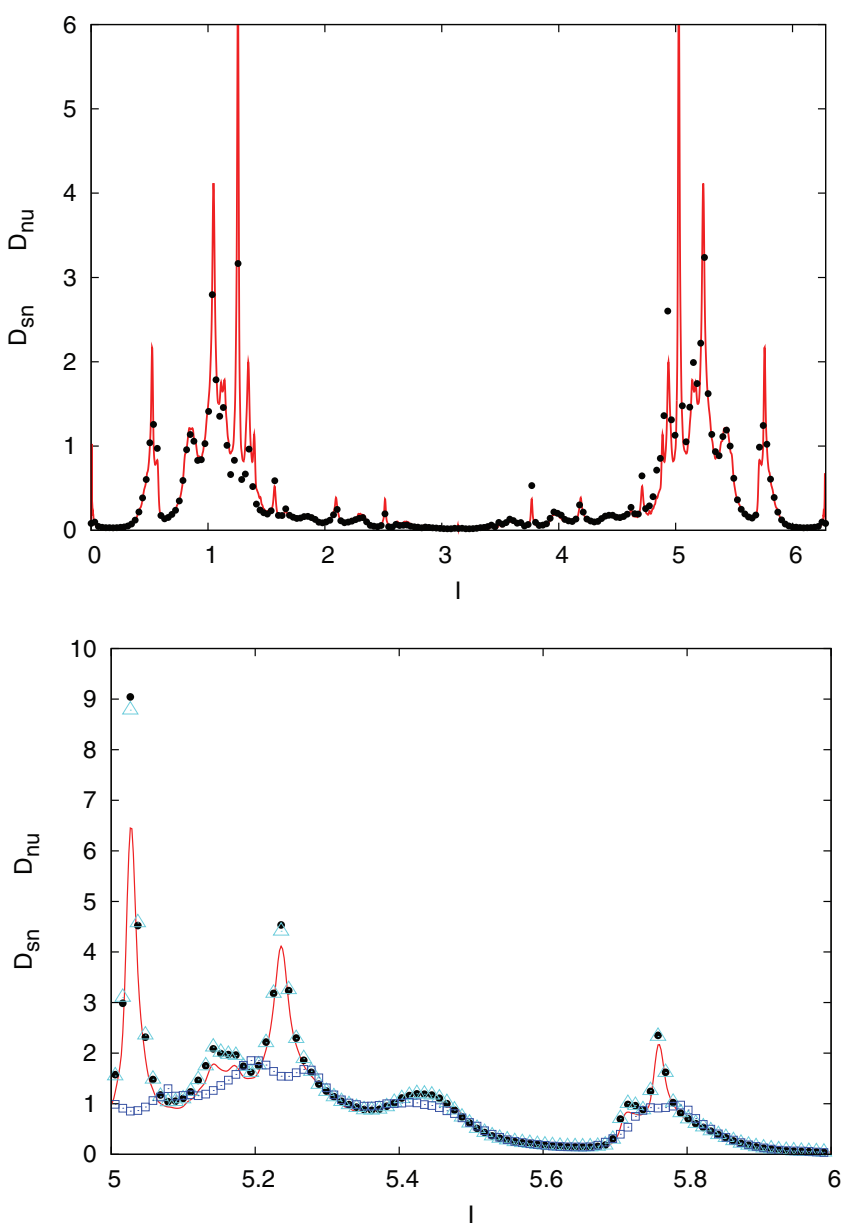

FIG. 13. (Color online) Diffusion coefficients for the symplectic map (1) for $K=0.9$ and $\mathcal{S}=\bigcup_{n=n_{S}}^{2 n_{s}} \mathcal{S}_{n}$ with $\mathcal{S}_{n}$ defined by Eq. (25). $\mathcal{D}_{s n}(I ; 500)$ is shown with a red solid line. $\mathcal{D}_{n u}(I ; 500)$ was computed for $\epsilon=10^{-3}, 10^{-5}, 10^{-7}$ and displayed with blue (square) dots, cyan (triangle-shaped) dots, and black (round) dots, respectively.

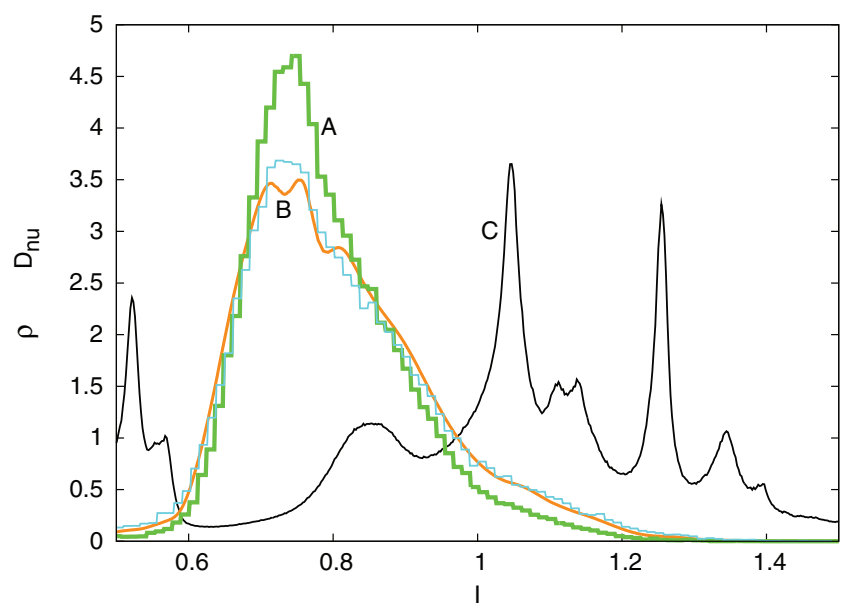

FIG. 14. (Color online) Distributions for thin layer regime ( $K=$ $0.9): \rho(I, 0.03), \rho_{\epsilon}\left(I, 3 \times 10^{4}\right)$, and $\rho_{\epsilon}\left(I, 5 \times 10^{4}\right)$, in colors orange (smooth line B), green (histogram A), and cyan (histogram B), respectively. For the histograms, the coupling parameter is $\epsilon=10^{-3}$. The black line $(\mathrm{C})$ displays the diffusion coefficient used when integrating the FP equation.

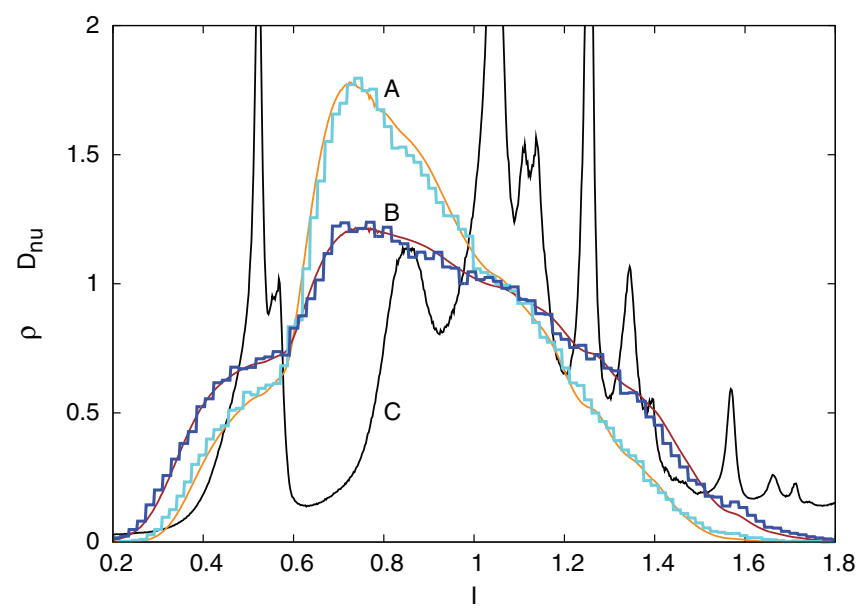

FIG. 15. (Color online) Distributions: $\quad \rho(I, 0.10), \quad \rho_{\epsilon}(I, 1.9 \times$ $\left.10^{5}\right), \rho(I, 0.19)$, and $\rho_{\epsilon}\left(I, 3.6 \times 10^{5}\right)$, in colors orange (smooth line A), cyan (histogram A), brown (smooth line B), and blue (histogram $\mathrm{B})$, respectively. For the histograms, the coupling parameter is $\epsilon=10^{-3}$. The black line (C) displays the diffusion coefficient used when integrating the FP equation.

evolved than, the histogram. In fact, the function that matches $\rho(I, 0.03)$ is $\rho_{\epsilon}\left(I, 5 \times 10^{4}\right)$, which is shown in cyan color (histogram B), so that $n=5 \times 10^{4}$ corresponds to $L=0.03$ and not to $L=0.05$. Thus, there is a shift of $\Delta L=0.02$ (for $L=0.03)$ in the diffusion time.

Figure 15 shows $\rho(I, 0.10), \rho_{\epsilon}\left(I, 1.9 \times 10^{5}\right), \rho(I, 0.19)$, and $\rho_{\epsilon}\left(I, 3.6 \times 10^{5}\right)$, in colors orange (smooth line A), cyan (histogram A), brown (smooth line B), and blue (histogram B), respectively. We can deduce that $\rho(I, 0.10)$ matches $\rho_{\epsilon}(I, 1.9 \times$ $\left.10^{5}\right)$ while $\rho(I, 0.19)$ does the same with $\rho_{\epsilon}\left(I, 3.6 \times 10^{5}\right)$. The first pair implies that $\Delta L=0.19-0.10=0.09$ for $L=0.10$ and the second pair implies that $\Delta L=0.36-0.19=0.17$ for $L=0.19$. As the value of $\Delta L$ is not constant for every time, we have that the relation between $L$ and $n$ can not be linear for this $K$ value. Neither is valid the $\epsilon$ dependence of $L$, as will be shown in Fig. 16. There, it can be seen that the histogram $\rho_{\epsilon}\left(I, 4 \times 10^{7}\right)$ for $\epsilon=10^{-4}$, shown in blue (A), does not match the concomitant histogram $\rho_{\epsilon}\left(I, 4 \times 10^{5}\right)$ for $\epsilon=10^{-3}$, shown in green (B). Instead, it resembles the histogram $\rho_{\epsilon}\left(I, 6.5 \times 10^{5}\right)$ for $\epsilon=10^{-3}$, displayed in cyan (C).

We conjecture that this discrepancy is the consequence of the slow correlation decaying in the chaotic dynamics with respect to the diffusion time scale $\simeq \epsilon^{-2}$ (see also the end of this section). Indeed, the numerical simulations point out that the amplitudes of the fluctuations of the autocorrelation functions decay much more slowly in the thin, than in the thick, layer case. This implies that for the smallest $K$ value the system is far away from the hypothesis of the (stochastic) averaging theorem. We suggest the possibility of a different scaling law between the original time and the diffusion time according to

$$
L=\epsilon^{2} n^{\alpha(\epsilon, K)}
$$

with $\alpha<1$ and $\lim _{\epsilon \rightarrow 0} \alpha(\epsilon, K)=1$ for $K$ values that correspond to sufficiently large chaotic layers. A simple numerical 


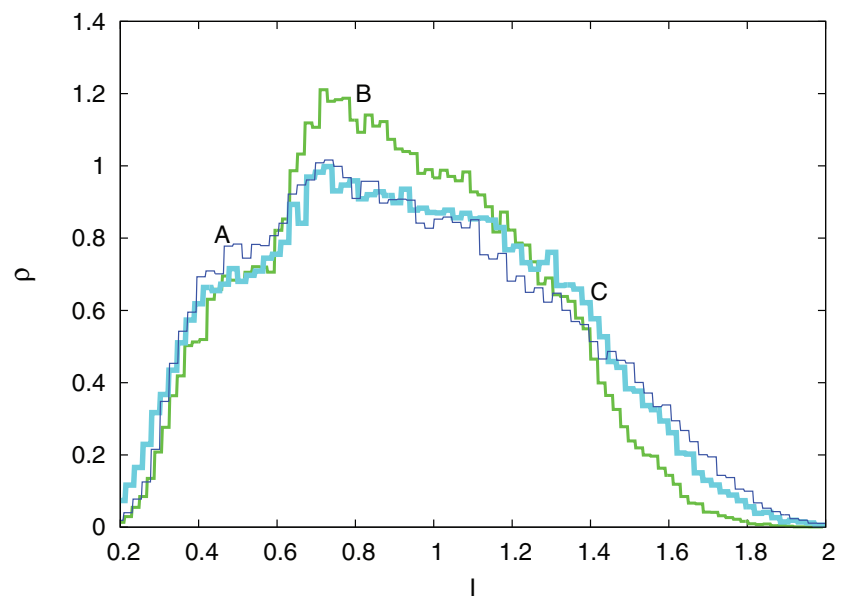

FIG. 16. (Color online) Histograms for thin layer regime $(K=$ $0.9): \rho_{\epsilon}\left(I, 4 \times 10^{7}\right) \quad\left(\epsilon=10^{-4}\right), \quad \rho_{\epsilon}\left(I, 4 \times 10^{5}\right) \quad\left(\epsilon=10^{-3}\right)$, and $\rho_{\epsilon}\left(I, 6.5 \times 10^{5}\right)\left(\epsilon=10^{-3}\right)$, in colors blue (A), green (B), and cyan (C), respectively.

interpolation from the numerical results with $\epsilon=10^{-3}$ gives $\alpha \simeq 0.95$, whereas for $\epsilon=10^{-4}$ we get $\alpha \approx 0.992$ (cf. Figs. 14, 15, and 16). This anomalous behavior in the diffusion dynamics is consistent with the local diffusion that can be observed in Fig. 8, where a power law with exponent $w \approx 0.92$ was fitted for $\epsilon=10^{-3}$ and $0 \leqslant L \leqslant 0.1$.

For both layer regimes, the persistence of correlations for every time is due to the existence of stability islands because the particles of the chaotic layer, when getting close enough to such islands, behave regularly, i.e., there the dynamics is "locally ordered."

Finally, let us look at the lower curve in Fig. 9, which represents the function $\sigma^{2}(L)$, for $0 \leqslant L \leqslant 2$ and $\epsilon=10^{-3}$. We infer that the global diffusion character depends also on the value of the parameter $K$ that determines different chaotic regimes. In the case of a thin chaotic layer, we remark that the variance evolution is not linear so that the global diffusion is anomalous. In the interval $0.5 \leqslant L \leqslant 2$ we have fitted a power law with exponent $w \approx 0.53$. This characterizes the macroscopic behavior as a subdiffusion. Notwithstanding, the global diffusion behavior is more complex than a power law and will be considered in a future work.

Moreover, in this case the backcoupling between the integrable and the chaotic degree of freedom produces a relevant effect on long-time iterations. In Fig. 17, we show a snapshot of the ensemble iterated with $\epsilon=10^{-3}$, at time $n=2 \times 10^{6}(L=2)$ and the comparison with Fig. 11 points out a non-negligible difference. It will be a subject of future works to quantify the influence of the backcoupling in the diffusion behavior, and try to figure out whether the dynamical origin of the anomalous diffusion is the backcoupling, or is an intrinsic property of the (uncoupled) SM noise, or it is due to the strong $I$ dependence of the diffusion coefficient. Nevertheless, the backcoupling has absolutely no effect in the computation of the diffusion coefficient, where the iteration time used is quite small, i.e., $N=500$. Plotting a snapshot of the ensemble at this time gives a distribution indistinguishable (so not shown) from the initial conditions from Fig. 11.

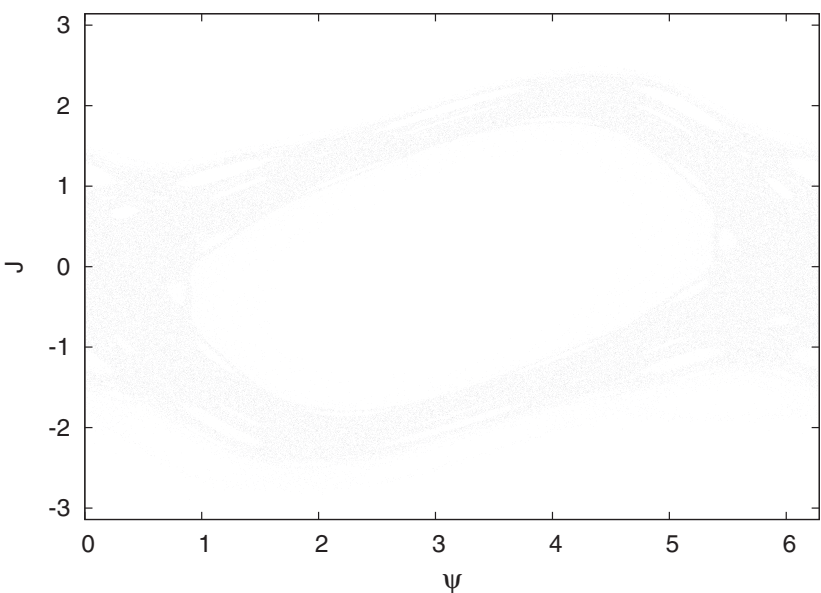

FIG. 17. Snapshot of the phase space $(J, \psi)$ for the map (1) computed after $2 \times 10^{6}$ iterations with $\epsilon=10^{-3}$ and $K=0.9$; i.e., projection onto the $[J, \psi]$ plane of the orbits associated to $\rho_{\epsilon}\left(I, 2 \times 10^{6}\right)$.

\section{CONCLUSION}

The main task developed in this article was the study of the diffusion behavior of a "quasiaction" I of a symplectic 4D a priori unstable map. As a tool to predict the diffusion coefficient, we have introduced a seminumerical method based on a theorem for stochastically perturbed Hamiltonian systems. This method allowed us to estimate the diffusion coefficient for two values of $K$, which correspond to the situations of thick and thin chaotic layer, and for a wide range of the perturbation parameter $\epsilon$ value. The results are consistent with an $\epsilon^{2}$ scaling of the diffusion coefficient which is the same dependence found in Refs. [32,56], also for an a priori unstable system.

We worked with chaotic test particles because the ensemble of orbits $\mathcal{S}$ was chosen in such a way that the initial conditions in the $[J, \psi]$ plane belonged to a chaotic layer. Even though for the symplectic map we did not use an analytic expression, the seminumerical diffusion coefficient is a prediction because it uses as input data only the unperturbed motion of the integrable part (the free rotator) and the numerical autocorrelation function of the perturbation (SM).

Regarding the behavior of the action distribution $\rho_{\epsilon}(I, n)$, we have found two different numerical results, depending on the chaotic regime. On the one hand, in the thick layer case, the distribution follows a FP equation. Besides, the time scaling relation $L=\epsilon^{2} n$, proposed in the stochastic theorem, between the (slow) FP time $L$, and the symplectic time $n$, turns out to be correct.

On the other hand, in the thin layer case, the distribution presents a "time delay" respect to the limit FP solution. This discrepancy is sensible because, in this regime, the SM "noise" is too far away from the conditions asked for in the stochastic theorem, mainly regarding the necessity that the autocorrelation function must decay to zero. We conjecture that if the correlation function is not decaying sufficiently fast with respect to the diffusion time scale $\epsilon^{-2}$, we have an anomalous scaling for fixed $K$ values, between the initial time and the diffusion time according to $L=\epsilon^{2} n^{\alpha(\epsilon, K)}$ where $\alpha<1$ 
and $\lim _{\epsilon \rightarrow 0} \alpha(\epsilon, K)=1$. This conjecture will be analyzed in a future work together with the character of global diffusion. In the thick layer regime, the diffusion behaved normally, while in the other case, it behaved subnormally.

The applicability of the presented stochastic approach goes beyond the particular 2.5 DoF Hamiltonian system chosen in this work. In particular, it would allow us to characterize and quantify diffusion in Hamiltonian systems that model perturbed simple nonlinear resonances.

We have also studied diffusion of a system built by coupling the free rotator with an ensemble of damped stochastic harmonic oscillators, finding a diffusion coefficient which differs considerably with respect to its deterministic counterpart. Moreover, we have justified why it is not possible to model a SM-driven diffusion with the one driven by a stochastic rotator.

In resume, we have given empirical evidence of the fact that a chaotic layer can act as a stochastic pump when coupled to some integrable system. After these results, we consider that the other way in which the chaotic layer is usually named after, i.e., stochastic layer, is phenomenologically suitable.

\section{ACKNOWLEDGMENTS}

We acknowledge the anonymous referees for their suggestions and constructive criticism, which helped to correct and improve this work. The stay of M. F. Mestre at the Physics Department of the University of Bologna was fully supported by a grant from the Erasmus Mundus External Cooperation Window Lot 16 Programme, EADIC, financed by the European Commission. Besides, P. M. Cincotta, C. M. Giordano, and M. F. Mestre were supported with grants from the Consejo Nacional de Investigaciones Científicas y Técnicas de la República Argentina (CONICET). This research has made use of NASA's Astrophysics Data System Bibliographic Services.

\section{APPENDIX A: STATIONARY AUTOCORRELATION FUNCTION OF THE DAMPED HARMONIC STOCHASTIC OSCILLATOR}

We will need two properties of the (Ito) stochastic integral of deterministic functions, that can be demonstrated from the basic definition found in elementary textbooks of the subject (for example, see Refs. [57,58]). These are the mean value formula

$$
E\left[\int_{t_{0}}^{t} f(z) d W_{z}\right]=0
$$

and the autocorrelation formula

$$
E\left[\int_{t_{0}}^{t} f(z) d W_{z} \int_{t_{0}}^{t^{\prime}} g(z) d W_{z}\right]=\int_{t_{0}}^{\min \left(t, t^{\prime}\right)} f(z) g(z) d z
$$

valid for arbitrary continuous functions $f(z)$ and $g(z)$ and arbitrary times $t$ and $t^{\prime}$.

From Eq. (17), the solution $\xi_{t}$ can be written as the sum of a deterministic term, denoted as $\xi_{t}^{(d)}$, and a stochastic one, denoted as $\xi_{t}^{(s)}$, according to the following definitions:

$$
\begin{aligned}
& \xi_{t}^{(d)}=\frac{\lambda \xi_{0}+2 v_{0}}{2 \omega_{1}} e^{-\frac{\lambda}{2} t} \sin \left(\omega_{1} t\right)+\xi_{0} e^{-\frac{\lambda}{2} t} \cos \left(\omega_{1} t\right), \\
& \xi_{t}^{(s)}=\frac{\sqrt{c}}{\omega_{1}} \int_{0}^{t} e^{-\frac{\lambda}{2}(t-z)} \sin \left[\omega_{1}(t-z)\right] d W_{z} .
\end{aligned}
$$

The mean value formula for $f(z) \equiv h(z ; t)=$ $e^{-\frac{\lambda}{2}(t-z)} \sin \left[\omega_{1}(t-z)\right]$ ( $t$ works as a parameter respect to the integral) implies that $E\left[\xi_{t}^{(s)}\right]=0$ so that

$$
E\left[\xi_{t} \xi_{t^{\prime}}\right]=\xi_{t}^{(d)} \xi_{t^{\prime}}^{(d)}+E\left[\xi_{t}^{(s)} \xi_{t^{\prime}}^{(s)}\right] .
$$

Considering also $g(z) \equiv h\left(z, t^{\prime}\right)$ and using the autocorrelation formula, we have that

$$
\begin{aligned}
E\left[\xi_{t}^{(s)} \xi_{t^{\prime}}^{(s)}\right]= & \frac{c}{\omega_{1}^{2}} \int_{0}^{\min \left(t, t^{\prime}\right)} h(z ; t) h\left(z ; t^{\prime}\right) d z \\
= & \frac{c}{\omega_{1}^{2}} \int_{0}^{\min \left(t, t^{\prime}\right)} e^{-\frac{\lambda}{2}\left(t+t^{\prime}-2 z\right)} \sin \left[\omega_{1}(t-z)\right] \\
& \times \sin \left[\omega_{1}\left(t^{\prime}-z\right)\right] d z .
\end{aligned}
$$

Integrating this expression and replacing $t^{\prime}=t+\tau$, for $\tau \geqslant 0$, we obtain that the stochastic contribution to the autocorrelation function is given by

$$
\begin{aligned}
E\left[\xi_{t}^{(s)} \xi_{t+\tau}^{(s)}\right]= & \frac{c}{4 \omega_{1}^{2}} e^{-\frac{\lambda}{2} \tau}\left\{\left(\frac{e^{i \omega_{1} \tau}+e^{-i \omega_{1} \tau}}{\lambda}\right)\left(1-e^{-\lambda t}\right)\right. \\
& -\frac{e^{i \omega_{1} \tau}}{\left(\lambda-i 2 \omega_{1}\right)}\left(1-e^{-\lambda t} e^{i 2 \omega_{1} t}\right) \\
& \left.-\frac{e^{-i \omega_{1} \tau}}{\left(\lambda+i 2 \omega_{1}\right)}\left(1-e^{-\lambda t} e^{-i 2 \omega_{1} t}\right)\right\} .
\end{aligned}
$$

Then, the following asymptotic behavior is satisfied:

$$
\begin{aligned}
& \lim _{t \rightarrow+\infty} E\left[\xi_{t}^{(s)} \xi_{t+\tau}^{(s)}\right] \\
& =\frac{c}{2 \lambda \omega^{2}} e^{-\frac{\lambda}{2} \tau}\left\{\cos \left(\omega_{1} \tau\right)+\frac{\lambda}{2 \omega_{1}} \sin \left(\omega_{1} \tau\right)\right\} .
\end{aligned}
$$

On the other side, it can be proven that the deterministic part does not contribute asymptotically because

$$
\lim _{t \rightarrow+\infty} \xi_{t}^{(d)} \xi_{t+\tau}^{(d)}=0 .
$$

From Eqs. (A1), (A2), and (A3), we obtain the desired expression

$$
\begin{aligned}
\phi_{S}(\tau) & \equiv \lim _{t \rightarrow+\infty} E\left[\xi_{t} \xi_{t+\tau}\right] \\
& =\frac{c}{2 \lambda \omega^{2}} e^{-\frac{\lambda}{2} \tau}\left\{\cos \left(\omega_{1} \tau\right)+\frac{\lambda}{2 \omega_{1}} \sin \left(\omega_{1} \tau\right)\right\} .
\end{aligned}
$$

\section{APPENDIX B: SPECTRAL DENSITY OF THE DAMPED HARMONIC STOCHASTIC OSCILLATOR}

According to the definition of the spectral density given in Eq. (9) and rewriting the trigonometric terms in the autocorrelation function with exponential functions, we obtain

$$
\begin{aligned}
& \tilde{\phi}_{s}(\nu) \\
& \quad=\frac{c}{4 \lambda \omega^{2}}\left\{\left(1-\mathrm{i} \frac{\lambda}{2 \omega_{1}}\right) G_{(+)}+\left(1+\mathrm{i} \frac{\lambda}{2 \omega_{1}}\right) G_{(-)}\right\},
\end{aligned}
$$


where we have introduced the series

$$
\begin{aligned}
G_{( \pm)} & =\sum_{m=-\infty}^{+\infty} \exp \left[-\frac{\lambda}{2}|m|+i\left(v m \pm \omega_{1}|m|\right)\right] \\
& =1+\sum_{m=1}^{+\infty}\left[p_{( \pm)}\right]^{m}+\sum_{m=1}^{+\infty}\left[q_{( \pm)}\right]^{m}
\end{aligned}
$$

with

$$
\begin{aligned}
& p_{( \pm)}=\exp \left[-\frac{\lambda}{2}+i\left( \pm \omega_{1}-v\right)\right] \\
& q_{( \pm)}=\exp \left[-\frac{\lambda}{2}+i\left( \pm \omega_{1}+v\right)\right] .
\end{aligned}
$$

As it happens that $\left|p_{( \pm)}\right|=\left|q_{( \pm)}\right|=e^{-\frac{\lambda}{2}}<1(\forall \lambda>0)$, the series converge to

$$
G_{( \pm)}=1+\frac{p_{( \pm)}}{1-p_{( \pm)}}+\frac{q_{( \pm)}}{1-q_{( \pm)}} .
$$

Introducing Eqs. (B2) and (B3) into (B1) and applying some arithmetical and trigonometrical properties, we arrive at a real- valued expression for the spectral density of the harmonic noise:

$$
\tilde{\phi}_{s}(v)=\frac{c}{4 \lambda \omega^{2}}\left[F_{1}\left(v ; \lambda, \omega_{1}\right)+\frac{\lambda}{2 \omega_{1}} F_{2}\left(v ; \lambda, \omega_{1}\right)\right],
$$

where the auxiliary functions $F_{k}(k=1,2)$ are given by

$$
\begin{aligned}
& F_{1} \equiv \frac{Z X_{(+)}+U_{(+)} Y_{(+)}}{\left|Q_{(+)}\right|^{2}}+\frac{Z X_{(-)}+U_{(-)} Y_{(-)}}{\left|Q_{(-)}\right|^{2}}, \\
& F_{2} \equiv \frac{Z Y_{(+)}+U_{(+)} X_{(+)}}{\left|Q_{(+)}\right|^{2}}-\frac{Z Y_{(-)}+U_{(-)} X_{(-)}}{\left|Q_{(-)}\right|^{2}},
\end{aligned}
$$

with

$$
\begin{aligned}
X_{( \pm)} \equiv & 1+e^{-\lambda} \cos \left(2 \omega_{1}\right) \\
& -e^{-\frac{\lambda}{2}}\left[\cos \left( \pm \omega_{1}-v\right)+\cos \left( \pm \omega_{1}+v\right)\right], \\
Y_{( \pm)} \equiv & \pm e^{-\lambda} \sin \left(2 \omega_{1}\right)-e^{-\frac{\lambda}{2}}\left[\sin \left( \pm \omega_{1}-v\right)+\sin \left( \pm \omega_{1}+v\right)\right], \\
Z \equiv & 1-e^{-\lambda} \cos \left(2 \omega_{1}\right), \\
U_{( \pm)} \equiv & \mp e^{-\lambda} \sin \left(2 \omega_{1}\right), \\
Q_{( \pm)} \equiv & X_{( \pm)}+\mathrm{i} Y_{( \pm)} .
\end{aligned}
$$

[1] A. Morbidelli, Regul. Chaotic Dyn. 6, 339 (2001).

[2] K. Tsiganis, in Topics in Gravitational Dynamics: Solar, ExtraSolar and Galactic Systems, Lecture Notes in Physics, Vol. 729, edited by D. Benest, C. Froeschle, and E. Lega (Springer, Berlin, 2007), p. 111.

[3] J. Laskar, Icarus 196, 1 (2008).

[4] H. Varvoglis, in IAU Colloq. 197: Dynamics of Populations of Planetary Systems, edited by Z. Knežević and A. Milani (Cambridge University Press, Cambridge, UK, 2005), pp. 157170.

[5] D. Nesvorný and A. Morbidelli, Astron. J. 116, 3029 (1998).

[6] A. Bazzani and H. Mais, in Nonlinear and Stochastic Beam Dynamics in Accelerators: A challenge to Theoretical and Computational Physics, DESY-PROC-1998-03, edited by A. Bazzani, J. Ellison, H. Mais, and G. Turchetti (Verlag Deutsches Elektronen-Synchrotron, Hamburg, Germany, 1998), pp. 74-84.

[7] H. Mais and M. P. Zorzano, Nuovo Cimento A 112-5, 467 (1999).

[8] R. Cogburn and J. A. Ellison, Commun. Math. Phys. 149, 97 (1992).

[9] T. Bountis and M. Kollmann, Phys. D (Amsterdam) 71, 122 (1994).

[10] G. Stancari, G. Annala, T. R. Johnson, D. A. Still, and A. Valishev, in Proceedings of IPAC2011, edited by C. Petit-JeanGenaz, A. Blanco, I. Etxebarria, F. Perez, A. Wolski, and V. Schaa (Joint Accelerator Conferences Website (JACoW), http://www.jacow.org/, 2011), pp. 1882-1884.

[11] N. N. Nekhoroshev, Russ. Math. Surv. 32, 1 (1977).

[12] B. V. Chirikov, Phys. Rep. 52, 263 (1979).

[13] M. Guzzo, E. Lega, and C. Froeschlé, Disc. Cont. Dyn. Syst. B 5, 687 (2005).

[14] C. Efthymiopoulos, Celest. Mech. Dynam. Astron. 102, 49 (2008).
[15] V. I. Arnold, Mathematical Methods of Classical Mechanics, 2nd ed. (Springer, New York, 1989).

[16] E. Lega, M. Guzzo, and C. Froeschlé, Phys. D (Amsterdam) 182, 179 (2003).

[17] C. Froeschlé, M. Guzzo, and E. Lega, Celest. Mech. Dyn. Astron. 92, 243 (2005).

[18] M. F. Mestre, P. M. Cincotta, and C. M. Giordano, in 3rd. La Plata International School on Astronomy and Geophysics: Chaos, Diffusion and Non-Integrability in Hamiltonian Systems - Aplications to Astronomy, edited by P. Cincotta, C. Giordano, and C. Efthymiopoulos, AAA Workshop Series Vol. 3 (Asociación Argentina de Astronomía and Universidad Nacional de La Plata, La Plata, Argentina, 2012), pp. 319-344.

[19] C. Efthymiopoulos and M. Harsoula, Phys. D (Amsterdam) 251, 19 (2013).

[20] P. M. Cincotta, C. Efthymiopoulos, C. M. Giordano, and M. F. Mestre, Physica D: Nonlinear Phenomena 266, 49 (2014).

[21] R. W. Easton, J. D. Meiss, and G. Roberts, Phys. D (Amsterdam) 156, 201 (2001).

[22] B. V. Chirikov, J. Ford, and F. Vivaldi, in Nonlinear Dynamics and the Beam-Beam Interaction, American Institute of Physics Conference Series, edited by M. Month and J. C. Herrera, Vol. 57 (AIP, New York, 1980), pp. 323-340.

[23] A. Morbidelli, Modern Celestial Mechanics: Aspects of Solar System Dynamics (Taylor \& Francis, Berlin, 2006).

[24] C. Efthymiopoulos, Celest. Mech. Dyn. Astron. 117, 101 (2013).

[25] J. L. Tennyson, M. A. Lieberman, and A. J. Lichtenberg, in Nonlinear Dynamics and the Beam-Beam Interaction, American Institute of Physics Conference Series, edited by M. Month and J. C. Herrera, Vol. 57 (AIP, New York, 1980), pp. 272-301.

[26] M. A. Lieberman, Ann. N. Y. Acad. Sci. 357, 119 (1980).

[27] G. R. Wang, B. Hu, and S. G. Chen, Phys. Lett. A 151, 37 (1990). 
[28] B. P. Wood, A. J. Lichtenberg, and M. A. Lieberman, Phys. Rev. A 42, 5885 (1990).

[29] K. Kaneko and R. J. Bagley, Phys. Lett. A 110, 435 (1985).

[30] S. Honjo and K. Kaneko, Geometric Structures of Phase Space in Multidimensional Chaos (Wiley, Hoboken, NJ, 2005), pp. 437-463.

[31] L. Chierchia and G. Gallavotti, Ann. I.H.P.: Phys. Theor. 60, 1 (1994).

[32] M. Guzzo, E. Lega, and C. Froeschlé, Commun. Math. Phys. 290, 557 (2009).

[33] P. Lochak, in Hamiltonian Systems with Three or More Degrees of Freedom, Series C: Math. and Phys. Sciences, Vol. 533, edited by C. Simó (Springer, Berlin, 1999).

[34] M. I. Freidlin and A. D. Wentzell, Random Perturbations of Dynamical Systems (Springer, New York, 1984).

[35] A. Bazzani, S. Siboni, and G. Turchetti, J. Phys. A: Math. Gen. 30, 27 (1997).

[36] W. Just, H. Kantz, C. Rödenbeck, and M. Helm, J. Phys. A: Math. Gen. 34, 3199 (2001).

[37] N. Baba, W. Just, H. Kantz, and A. Riegert, Phys. Rev. E 73 066228 (2006).

[38] A. Riegert, W. Just, N. Baba, and H. Kantz, Phys. Rev. E 76, 066211 (2007).

[39] I. Melbourne and A. M. Stuart, Nonlinearity 24, 1361 (2011).

[40] G. A. Gottwald and I. Melbourne, Proc. R. Soc. London, Ser. A 469, 20130201 (2013).

[41] A. J. Lichtenberg and M. A. Lieberman, Regular and Chaotic Dynamics, 2nd ed. (Springer, New York, 1992).

[42] H. Varvoglis, in Hamiltonian Systems and Fourier Analysis: New Prospects for Gravitational Dynamics, edited by
D. Benest, C. Froeschle, and E. Lega,Advances in Astronomy and Astrophysics (Cambridge University Press, Cambridge, UK, 2005), Chap. 6

[43] Y. Kominis, A. K. Ram, and K. Hizanidis, Phys. Rev. Lett. 104, 235001 (2010).

[44] G. M. Zaslavsky, Phys. Rep. 371, 461 (2002).

[45] H.-T. Kook and J. D. Meiss, Phys. Rev. A 41, 4143 (1990).

[46] J. R. Cary, J. D. Meiss, and A. Bhattacharjee, Phys. Rev. A 23, 2744 (1981).

[47] A. Bazzani and L. Beccaceci, J. Phys. A: Math. Gen. 31, 5843 (1998).

[48] R. Z. Khasminskii, Theory Probab. Its Appl. 11, 390 (1966).

[49] A. Einstein, in Investigations on the Theory of the Brownian Movement, edited by R. Furth (Dover, New York, 1956), pp. $1-18$.

[50] G. E. Uhlenbeck and L. S. Ornstein, Phys. Rev. 36, 823 (1930).

[51] S. Chandrasekhar, Rev. Mod. Phys. 15, 1 (1943).

[52] M. C. Wang and G. E. Uhlenbeck, Rev. Mod. Phys. 17, 323 (1945).

[53] S. F. Nørrelykke and H. Flyvbjerg, Phys. Rev. E 83, 041103 (2011).

[54] D. T. Gillespie, Am. J. Phys. 64, 225 (1996).

[55] A. Shalchi, Phys. Rev. E 83, 046402 (2011).

[56] E. Lega, M. Guzzo, and C. Froeschlé, Celest. Mech. Dynam. Astron. 107, 115 (2010).

[57] P. R. Gardiner, Handbook of Stochastic Methods (Springer, Berlin, 2004).

[58] L. Arnold, Stochastic Differential Equations: Theory and Applications (Wiley, New York, 1973). 